\title{
FORM FACTORS, DEFORMED KNIZHNIK-ZAMOLODCHIKOV EQUATIONS AND FINITE-GAP INTEGRATION.
}

\author{
Fedor A. Smirnov \\ Isaac Newton Institute, Cambridge, CB3 OEH, U.K. \\ and \\ Steklov Mathematical Institute, St. Petersburg 191011, RUSSIA
}

\begin{abstract}
We study the limit of asymptotically free massive integrable models in which the algebra of nonlocal charges turns into affine algebra. The form factors of fields in that limit are described by $\mathrm{KZ}$ equations on level 0 . We show the limit to be connected with finite-gap integration of classical integrable equations.
\end{abstract}

\section{Motivations.}

The nonlocal symmetries of integrable models of quantum field theory in two dimensions were first studied several years ago [1]. The reason for that being in attempts to understand the quantization of asymptotically free models. Being almost forgotten for some time the nonlocal symmetries returned to the field rather indirectly, namely, through the finite-dimensional quantum group symmetries of CFT. Now it is understood that the integrability of massive models is closely connected with possessing infinite-dimensional algebra of nonlocal symmetries [2-6]. The local integrals constitute a center of it. The algebra of nonlocal symmetries is always a Hopf algebra, particles transform under its finite-dimensional representations while quasilocal fields constitute infinite-dimensional multiplets with highest vectors corresponding to the local fields. The S-matrix is nothing but universal R-matrix specified onto finite-dimensional representations while the braiding of the multiplets of quasilocal fields is described by the universal R-matrix specified onto the tensor product of two Verma modules. Moreover, it was shown [6] that the form factors which put together particles and fields can be considered as solutions of deformed Knizhnik-Zamolodchikov equations [7] which is quite natural since these equations use to relate finite-dimensional representations of deformed loop algebras with infinite-dimensional highest weight representations.

A question which should be asked is the following. Suppose we have an integrable model with symmetry under certain deformation of the loop algebra. Then what is the meaning of the "classical limit" which moves the deformed algebra into undeformed one. The answer to this question is not trivial as we shall see. For many models this limit does not look to make much sense. The point is that the limit is related not to the rescaling of space coordinates (or momenta) as conformal limit is, but rather to rescaling of rapidities of particles which are logarithms of momenta. In a sense the limit in question is even opposite to conformal limit in 
which we throw away all the logarithms preserving powers, here we preserve logarithms, but throw away powers considering the asymptotic expansions. However, the limit seems to be reasonable for asymptotically free models. So, we return to the same idea as years ago: to use nonlocal symmetries in asymptotically free theories, certainly we return to this idea with new experience. It should be said also that there exist certain problems with correspondence between local integrals of motion for classical and quantum cases in the theory of asymptotically free models. The reason for that is very fundamental, that is why our consideration will be not Hamiltonian one.

Particular (and, probably, personal) interest in this business is the following. We have very nice formulae for final physical quantities in both classical and quantum theory of integrable models. However existing way of quantization [8](which has clear Hamiltonian meaning) does not allow to proceed directly from ones to others, it starts with beautiful things (R-matrix, algebraic Bethe anzatz), but then leads through usual jungle with renormalizations and all that. We would like to have direct way even if it has less clear meaning. Similar ideas were applied to study of correlation functions of vertex models of statistical mechanics in recent works from Kyoto school [9].

Let us consider a particular asymptotically free model. Namely, let it be the $s u(2)$ chiral Gross-Neveu model. The spectrum of the model contains one twocomponent particle. The two-particle S-matrix is given by [10]:

$$
S_{1,2}\left(\beta_{1}-\beta_{2}\right)=\frac{\Gamma\left(\frac{\beta_{1}-\beta_{2}}{2 \pi i}\right) \Gamma\left(\frac{1}{2}+\frac{\beta_{1}-\beta_{2}}{2 \pi i}\right)}{\Gamma\left(\frac{\beta_{1}-\beta_{2}}{2 \pi i}\right) \Gamma\left(\frac{1}{2}-\frac{\beta_{1}-\beta_{2}}{2 \pi i}\right)}\left[\frac{\left(\beta_{1}-\beta_{2}\right)-\pi i P_{1,2}}{\beta_{1}-\beta_{2}-\pi i}\right],
$$

where $P_{1,2}$ is permutation. If we are interested in local operators which transform under integer-spin representation of global $S L(2)$ isotopic group all of them can be obtained as descendents with respect to different conservation laws of one nonlocal operator called $\mathfrak{A}\left(x_{0}, x_{1}\right)$. The connection with important local operators will be explained later. The operator $\mathfrak{A}\left(x_{0}, x_{1}\right)$ is scalar with respect to both isotopic and Lorenz transformations. In form factor approach [11] it can be presented as follows:

$$
\begin{aligned}
& \mathfrak{A}\left(x_{0}, x_{1}\right)=\sum_{m, n=0}^{\infty} \frac{1}{m ! n !} \\
& \times \int_{-\infty}^{\infty} d \alpha_{1} \cdots \int_{-\infty}^{\infty} d \alpha_{m} \int_{-\infty}^{\infty} d \beta_{1} \cdots \int_{-\infty}^{\infty} d \beta_{n} Z_{\epsilon_{1}}^{*}\left(\alpha_{1}\right) \cdots Z_{\epsilon_{m}}^{*}\left(\alpha_{m}\right) \\
& \left.\times F\left(\alpha_{m}, \cdots, \alpha_{1} \mid \beta_{1}, \cdots, \beta_{n}\right)_{\delta_{1}, \cdots, \delta_{n}}^{\epsilon_{m}, \cdots} Z^{\delta_{n}}\left(\beta_{n}\right) \cdots Z^{\delta_{1}} \beta_{1}\right) \\
& \times \exp \left(\sum i p^{\mu}\left(\alpha_{i}\right) x_{\mu}-\sum i p^{\mu}\left(\beta_{i}\right) x_{\mu}\right)
\end{aligned}
$$

where $Z^{*}, Z$ are Zamolodchikov-Faddeev creation-annihilation operators of particles, $p_{\mu}(\alpha)$ is one-particle energy-momentum $\left(p_{\mu}(\alpha)=M\left(\exp (\alpha)+(-1)^{\mu} \exp (\alpha)\right)\right)$, $\epsilon, \delta= \pm$ are isotopic indices. $F$ are form factors. They satisfy the crossing symmetry requirement:

$$
\begin{aligned}
& F\left(\alpha_{m}, \cdots, \alpha_{1} \mid \beta_{1}, \cdots, \beta_{n}\right)_{\delta_{1}, \cdots, \delta_{n}}^{\epsilon_{m}, \cdots, \epsilon_{1}}=c_{\delta_{1}, \delta_{1}^{\prime}} \cdots c_{\delta_{n}, \delta_{n}^{\prime}} \\
& \times F\left(\alpha_{m}, \cdots, \alpha_{1}, \beta_{1}+\pi i-i 0, \cdots, \beta_{n}+\pi i-i 0\right)^{\epsilon_{m}, \cdots, \epsilon_{1}, \delta_{1}^{\prime}, \cdots, \delta_{n}^{\prime}}, c=i \sigma^{2},
\end{aligned}
$$


It should be mentioned that for the operator $\mathfrak{A}$ the two particle form factor has a double pole at $\beta_{2}=\beta_{1}+\pi i$ the corresponding singular part being normalized as $\left(\beta_{2}-\beta_{1}-\pi i\right)^{-2} c^{\epsilon_{1}, \epsilon_{2}}$.

The model allows abelian and non-abelian symmetries. The first are the local conservation laws with all possible odd spins. The one particle eigenvalues of the local integrals $I_{s}(s=2 m+1, m=-\infty, \cdots, \infty)$ are equal to $\exp (s \alpha)$ the spectrum of them being additive which can be expressed in more formal way by the following comultiplication formula:

$$
\Delta\left(I_{s}\right)=I_{s} \otimes 1+1 \otimes I_{s}
$$

The nonlocal symmetries together with $S L(2)$-charges constitute the Yangian $(Y)$ [2]. The generators of $Y$ are $J_{0}^{a}, J_{1}^{a}, a=1,2,3$ whose action onto one particle is described by $\sigma^{a}, \alpha \sigma^{a}$ respectively and the comultiplication is given by

$$
\begin{aligned}
& \Delta\left(J_{0}^{a}\right)=J_{0}^{a} \otimes 1+1 \otimes J_{0}^{a}, \\
& \Delta\left(J_{1}^{a}\right)=J_{1}^{a} \otimes 1+1 \otimes J_{1}^{a}+\pi i f^{a b c} J_{0}^{b} \otimes J_{0}^{c}
\end{aligned}
$$

Every operator acting in the space of states $\left(H_{p}\right)$ which is the Fock space of particles has descendents with respect to both local and non-local symmetries defined through ajoint action. One can think of the space $H_{p}$ as of

$$
H_{p}=\bigoplus_{n=1}^{\infty} \int_{\beta_{1}<\cdots<\beta_{2 n}} V_{\beta_{1}} \otimes \cdots \otimes V_{\beta_{2 n}}
$$

where $V_{\beta_{i}}$ is the space of the representation $\rho_{\beta_{i}}$. The operators $Z^{*}(\beta), Z(\beta)$ either add or remove one space $V_{\beta}$. In particular the energy-momentum tensor and currents are the following descendents of the operator $\mathfrak{A}$ :

$$
T_{\mu, \nu}\left(x_{0}, x_{1}\right)=\epsilon_{\mu, \mu^{\prime}} \epsilon_{\nu, \nu^{\prime}} P_{\mu^{\prime}} P_{\nu^{\prime}}\left(\mathfrak{A}\left(x_{0}, x_{1}\right)\right), j_{\mu}^{a}\left(x_{0}, x_{1}\right)=\epsilon_{\mu, \mu^{\prime}} P_{\mu^{\prime}} J_{1}^{a}\left(\mathfrak{A}\left(x_{0}, x_{1}\right)\right)
$$

where $P_{0}=I_{1}+I_{-1}, P_{1}=I_{1}-I_{-1}$. There are more general relations. For example the density of the local conservation law $I_{s}\left(d_{s}\left(x_{0}, x_{1}\right)\right)$ is obtained in the following way:

$$
d_{s}\left(x_{0}, x_{1}\right)=P_{0} I_{s}\left(\mathfrak{A}\left(x_{0}, x_{1}\right)\right)
$$

The last relation is very much in common with the classical relation identifying the densities of local conservation laws with the derivatives of logarithm of $\tau$-function. So, it is reasonable to think of the operator $\mathfrak{A}\left(x_{0}, x_{1}\right)$ as of the quantum analog of $\log (\tau)$. Certainly, this is a formal analogy and in order to make it more instructive we have to develop the relation with the classical $\tau$-function. This is the main goal of the present paper. For the reasons which will be explained later the connection in question can hardly be achieved in Hamiltonian formalism, so, our strategy will be to find a "good" formula in classics in which all the objects involved in quantum formula (the operator $\mathfrak{A}$, the form factors, Zamolodchikov-Faddeev operators) will have their classical analogs. This is what we are going to do in that paper. But before proceeding in that direction we have to remind certain facts about the symmetries of the quantum model. 


\section{The Yangian symmetry.}

In this section the brief account of the recent developments concerning the dynamical symmetries of integrable models will be presented.

As it has been already mentioned the model under consideration allows nonlocal conservation laws $J_{1}^{a}$ which together with isotopic charges $J_{0}^{a}$ constitute the infinite dimensional algebra called Yangian [12]. It is explained in $[5,6]$ that for the application to the description of local and quasilocal operators in the theory one has to add one more generator $\left(J_{-1}^{a}\right)$ to the algebra. The full algebra generated by $J_{0}^{a}, J_{1}^{a}, J_{-1}^{a}$ is called the double of Yangian being denoted by $D(Y)$. One can think of $D(Y)$ as of a deformation of the affine algebra $\widehat{s l}(2)$ with natural identification of the generators. This will be explained more precisely later. The Yangian $Y$ is a subalgebra of $D(Y)$ which acting onto the fields in the theory creates descendents. The introducing of the second half of the algebra was originally motivated by the problem of description of the commutation relations among these descendents[5]. The commutation relations are described as follows. Consider a local operator $\varphi\left(x_{0}, x_{1}\right)$ which can be for the simplicity taken as invariant under the isotopic algebra. Now create all possible descendents of the operator acting on it by arbitrary number of the operators $J_{1}^{a}$ :

$$
\varphi\left(x_{0}, x_{1}\right)^{a_{1}, \cdots, a_{n}}=J_{1}^{a_{1}} \cdots J_{1}^{a_{n}}\left(\varphi\left(x_{0}, x_{1}\right)\right)
$$

We can combine these operators into $\Phi$ considering $a_{1}, \cdots, a_{n}$ as multiindex. So, $\Phi$ is an infinite column of operators which can be considered as belonging to $\operatorname{End}\left(H_{p}\right) \otimes W$ where $H_{p}$ is the space of states, $W$ is the Verma module created by action of the operator $J_{1}^{a}$. Now, if we consider two towers of operators $\Phi\left(x_{0}, x_{1}\right)$ and $\Phi\left(x_{0}, x_{1}^{\prime}\right)$ all the products of their elements can be combined in the following object: $\Phi_{1}\left(x_{0}, x_{1}\right) \Phi_{2}\left(x_{0}, x_{1}^{\prime}\right)$ where two copies of $W\left(W_{1}, W_{2}\right)$ are considered and $\Phi_{1} \in \operatorname{End}\left(H_{p}\right) \otimes W_{1}, \Phi_{2} \in \operatorname{End}\left(H_{p}\right) \otimes W_{2}$. The commutation relation in question can be written down in the following way [5]:

$$
\Phi_{1}\left(x_{0}, x_{1}\right) \Phi_{2}\left(x_{0}, x_{1}^{\prime}\right)=R_{1,2} \Phi_{1}\left(x_{0}, x_{1}^{\prime}\right) \Phi_{2}\left(x_{0}, x_{1}\right), \text { for } x_{1}>x_{1}^{\prime}
$$

Here $R_{1,2}$ is $D(Y)$ R-matrix acting in the tensor product of two Verma moduli. That means that in these relations we effectively consider the local field $\varphi$ as that annihilated by the operator $J_{-1}^{a}$.

It is explained in the papers [6] that the situation can be inversed. We considered the space of particles and came to the conclusion that the local and quasilocal operators acting in the space behave, as far as the commutation relations are considered, as being combined into Verma moduli. The local operators themselves are identified with the highest vectors of the Verma moduli. We can consider now the space of fields instead of the space of particles. The space of fields $\left(H_{f}\right)$ is a suitable collection of Verma moduli. Particular local field $\varphi_{i}$ is identified with a highest vector $|0\rangle_{i}$ satisfying the requirement

$$
J_{-1}^{a}|0\rangle_{i}=0
$$

We consider also a dual vacuum $\langle 0|$ which is defined by the relations

$$
\langle 0| J_{0}^{a}=0,\langle 0| J_{1}^{a}=0
$$


The algebra $D(A)$ allows beside of the infinite dimensional representations the finite-dimensional ones. We will be interested in two-dimensional representation $\left(\rho_{\beta}\right)$ depending on a parameter $\beta$, the detailed description is given in [6]. One can define the vertex operators $V^{\epsilon}(\beta)$ which belongs to $\operatorname{End}\left(H_{f}\right) \otimes C^{2}\left(\epsilon= \pm\right.$ is $C^{2}$ index). Acting in $H_{f}$ the operator $V^{\epsilon}(\beta)$ transforms under the ajoint representation with respect to the representation $\rho_{\beta}$ :

$$
a d_{x}\left(V^{\epsilon}(\beta)\right)=\rho_{\beta}(x)_{\epsilon^{\prime}}^{\epsilon} V^{\epsilon^{\prime}}(\beta)
$$

Then the vacuum expectations of the operators $V^{\epsilon}(\beta)$ given by

$$
\left\langle 0\left|V^{\epsilon_{1}}\left(\beta_{1}\right) \cdots V^{\epsilon_{2 n}}\left(\beta_{2 n}\right)\right| 0\right\rangle_{i}
$$

satisfy the deformed Knizhnik-Zamolodchikov equations, in usual tensor notation they take a form:

$$
\begin{aligned}
& \left\langle 0\left|V\left(\beta_{1}\right) \cdots V\left(\beta_{j}+2 \pi\right) \cdots V\left(\beta_{2 n}\right)\right| 0\right\rangle_{i}= \\
& =S_{2 n, j}\left(\beta_{2 n}-\beta_{j}-2 \pi i\right) \cdots S_{j+1, j}\left(\beta_{j+1}-\beta_{j}-2 \pi i\right) \\
& \times S_{1, j}\left(\beta_{1}-\beta_{j}\right) \cdots S_{j-1, j}\left(\beta_{j-1}-\beta_{j}\right) \\
& \times\left\langle 0\left|V\left(\beta_{1}\right) \cdots V\left(\beta_{j}\right) \cdots V\left(\beta_{2 n}\right)\right| 0\right\rangle_{i}
\end{aligned}
$$

As it has been explained in [12] these equations are consistent with the symmetry property

$$
\begin{aligned}
& S_{i, i+1}\left(\beta_{i}-\beta_{i+1}\right)\left\langle 0\left|V\left(\beta_{1}\right), \cdots, V\left(\beta_{i}\right), V\left(\beta_{i+1}\right), \cdots, V\left(\beta_{2 n}\right)\right| 0\right\rangle_{i}= \\
& =P_{i, i+1}\left\langle 0\left|V\left(\beta_{1}\right), \cdots, V\left(\beta_{i+1}\right), V\left(\beta_{i}\right), \cdots, V\left(\beta_{2 n}\right)\right| 0\right\rangle_{i}
\end{aligned}
$$

Being supplied with the symmetry property (2.2) the equations (2.1) appear to be the same as the basic requirements for the form factors. There is an additional equation on residues in the form factor bootstrap approach [11] which we do not present here. This equation can be interpreted as a form of operator product requirement [6]. It is responsible for the special choice of blocks of the vertex operators. Namely, the following remarkable identification can be done

$$
F\left(\beta_{1}, \cdots, \beta_{2 n}\right)^{\epsilon_{1}, \cdots, \epsilon_{2 n}} \sim\left\langle 0\left|V^{\epsilon_{1}}\left(\beta_{1}\right) \cdots V^{\epsilon_{2 n}}\left(\beta_{2 n}\right)\right| 0\right\rangle
$$

where $|0\rangle$ is the highest vector of spin zero, The consequence of the Verma moduli in the RHS is taken as follows (we indicate the spins of the highest vectors)

$$
0 \rightarrow 1 / 2 \rightarrow 1 \rightarrow 1 / 2 \rightarrow 1 \rightarrow \cdots \rightarrow 1 / 2 \rightarrow 0
$$

We put the asymptotical equivalence in (2.3) for the reasons explained in [6], we shall briefly explain the point later. The form factor can be considered as a matrix element of the operator $\mathfrak{A}$ in the space of particles. More generally the relation (2.3) can be rewritten as follows

$$
\left\langle v a c\left|Z^{\epsilon_{1}}\left(\beta_{1}\right) \cdots Z^{\epsilon_{2 n}}\left(\beta_{2 n}\right) \varphi_{i}(0)\right| v a c\right\rangle \sim\left\langle 0\left|V^{\epsilon_{1}}\left(\beta_{1}\right) \cdots V^{\epsilon_{2 n}}\left(\beta_{2 n}\right)\right| 0\right\rangle_{i}
$$


We do not expect other local operators then the descendents under the action of local integrals on $\mathfrak{A}$, and on the currents $j_{\mu}^{a}$ to exist in the theory. There is also parafermionic field of spin $\frac{1}{4}$ with respect to Lorentz transformations (kink field). From the point of view of the representation theory these three fields correspond to spin $0,1,1 / 2$ highest vectors respectively. The sequence of the Verma moduli in form factors of these operators is the same as in (2.4) the only difference being that the sequence for currents (kinks) terminates at spin $1(1 / 2)$ representation. This is a variant of "rationality" of the theory. The reason for the phenomenon to occur supposed to be in the absence of mixing of different solution of deformed KZ equation by braiding which is discussed in details in the papers [6].

Now we are going to explain the asymptotical character of the equivalence in the relations (2.3). This matter was considered in [6,12]. The situation is briefly as follows. In LHS of (2.3) we have the analytical solutions of (2.1) while the RHS contains the vacuum expectation of $D(Y)$-vertex operators. The RHS can be in principle calculated directly (without use of KZ) using the definition of the vertex operators. It is quite clear that the only thing we are able to get in that way are certain power series in $\beta$ 's. But as it follows from the explicit formulae for the analytical solutions they are transcendental functions of $\beta$ 's (the explicit formulae are given in [11]); roughly, they have the properties of $\Gamma$-functions. The only reasonable connection between these two types of objects is asymptotical one. One can have in mind the following analogy: $\Gamma$-function (RHS) and its asymptotical series (LHS) both satisfy the functional equation for the $\Gamma$-function (dKZ-equation). The difference between the function and its asymptotics in the case is due to the exponential in $\beta$ contributions which are considered as the contribution due to the intermediate state created by local integrals which should be added to the $D(Y)$ $[6]$. 


\section{The classical limit.}

As it has been already said the Yangian double is a deformation of the algebra $\widehat{s l}(2)$. Let us ignore for the moment the physical content, and consider the formal aspects of the limit. The typical relation for us will be (1.2). By rescaling of $J_{1}^{a}$ the relation can be rewritten as

$$
\begin{aligned}
& \Delta\left(J_{0}^{a}\right)=J_{0}^{a} \otimes 1+1 \otimes J_{0}^{a}, \\
& \Delta\left(J_{1}^{a}\right)=J_{1}^{a} \otimes 1+1 \otimes J_{1}^{a}+\frac{h}{2} f^{a b c} J_{0}^{b} \otimes J_{0}^{c}
\end{aligned}
$$

where $h$ is arbitrary constant. Certainly, the generator $J_{-1}^{a}$ should be rescaled as well. Now consider $h$ as Planck constant and take the limit $h \rightarrow+i 0$ ( we prefer considering imaginary $h$, so, probably, temperature is better analog for it then Planck constant). In the limit the equations (3.1) turn into trivial comultiplication formulae for the generators of $\widehat{s l}(2)$, all other $D(Y)$-relations turn into $\widehat{s l}(2)$-ones in the limit. Also the dKZ equations turn formally into usual $\widehat{s l}(2) \mathrm{KZ}$ equations on level zero. It is clear that the rescaling of the generators we did for the $\mathrm{dKZ}$ equations is equivalent to the rescaling of the rapidities: $\beta_{i}=\frac{2 \pi i}{h} \lambda_{i}, h \rightarrow+i 0$ while $\lambda_{i}$ are fixed. So, formally for the functions

$$
f\left(\lambda_{1}, \cdots, \lambda_{2 n}\right) \simeq_{h \rightarrow+i 0} C(h) F\left(\frac{2 \pi i \lambda_{1}}{h}, \cdots, \frac{2 \pi i \lambda_{2 n}}{h}\right)
$$

$(C(h)$ is a normalization constant) the equations (2.1) turn in this limit into

$$
\left(\frac{d}{d \lambda_{i}}+\sum_{i \neq j} r_{i, j}\left(\lambda_{i}-\lambda_{j}\right)\right) f\left(\lambda_{1}, \cdots, \lambda_{2 n}\right)=0
$$

where $r$ is the classical r-matrix:

$$
\left.r_{i, j}\left(\lambda_{i}-\lambda_{j}\right)\right)=\frac{\sigma_{i}^{a} \otimes \sigma_{j}^{a}}{\lambda_{i}-\lambda_{j}}
$$

The connection between deformed and undeformed KZ equation is described explicitly in [13]. Let us outline the basic points.

First, the dKZ equation allows the same number of solutions as undeformed one. The solution corresponding to the form factors is a special one. Its particular character is explained by (2.4), we shall also describe it from other point of view soon.

Second, the solutions are in one-to-one correspondence in the following sense. Consider some solution of $\mathrm{dKZ}$ for $\beta_{1}<\beta_{2}<\cdots<\beta_{2 n}$ then its asymptotics $\beta_{i}=\frac{2 \pi i}{h} \lambda_{i}, h \rightarrow+i 0$ is described by a solution of KZ. In the paper [13] the explicit formulae are presented for solution of dKZ which correspond to all possible solutions of $\mathrm{KZ}$ in that sense.

Third, there is essential difference in the properties of the solution of dKZ in comparison with those of KZ: braiding does not mix different solutions of dKZ. The difference is explained by the asymptotical character of the correspondence above. Braiding does not commute with taking the asymptotics.

The third point mentioned makes a real difference between the deformed and underformed case. In particular, in the deformed case it makes sense to consider 
the form factors of $\mathfrak{A}$ themselves without consideration other solutions to dKZ equations. But taking the asymptotics of the the form factors we have to consider not only the solution of $\mathrm{KZ}$ we obtain, but also those which are connected with it by braiding. We shall call this part of solutions of $\mathrm{KZ}$ the main part.

We do not give here the explicit formulae for the solutions of dKZ which can be found in $[11,13]$, but we have to write down explicitly the solutions of $\mathrm{KZ}$. These formulae concerns the particular case of zero central extension which causes essential simplification with respect to the general case. So, let us explain first how the simplifications appear. In Varchenko-Shechtman [14] formulae for the solutions of $\mathrm{KZ}$ one has the following structure:

$$
\begin{aligned}
\prod_{i<j}\left(\lambda_{i}-\lambda_{j}\right)^{\frac{1}{2(k+2)}} \int d \tau_{1} \cdots d \tau_{k} & \prod_{m, i}\left(\tau_{m}-\lambda_{i}\right)^{\frac{1}{(k+2)}} \prod_{l<m}\left(\tau_{l}-\tau_{m}\right)^{\frac{2}{(k+2)}} \\
& \times R\left(\tau_{1}, \cdots ; \lambda_{1}, \cdots\right)
\end{aligned}
$$

where $\tau$ 's are integration variables (corresponding to screenings), $R$ is a rational function of its variables. For $k=0$ the exponents in (3.2) turn into $1 / 4,1 / 2,1$ respectively. The first one is not so essential, the second causes the hyperelliptic character of the integrals, the third causes the absence of branching between the integration variables which simplifies essentially the choice of contours of integration. Also one can present $\prod_{l<m}\left(\tau_{l}-\tau_{m}\right)$ as Vandermond determinant, and rewriting properly the function $R$ perform the integration over the columns of the determinant getting a determinant of single integrals instead of the multi- integral of determinant. Let us present the result to which these manipulations should lead (which actually has not been obtained in that way, but directly from the classical limit the solutions of $\mathrm{dKZ}$ solutions).

Consider the components of $f\left(\lambda_{1}, \cdots, \lambda_{2 n}\right): f\left(\lambda_{1}, \cdots, \lambda_{2 n}\right)^{\epsilon_{1}, \cdots, \epsilon_{2 n}}$. We are looking for the singlet solutions of the equations which means in particular that $\sum \epsilon_{i}=0$. For each particular component $f\left(\lambda_{1}, \cdots, \lambda_{2 n}\right)^{\epsilon_{1}, \cdots, \epsilon_{2 n}}$ the multiindex $\epsilon_{1}, \cdots, \epsilon_{2 n}$ induces a partition of $B=\{1, \cdots, 2 n\}$ into $T=\left\{i_{k}\right\}_{k=1}^{n}: \epsilon_{i_{k}}=+$ and $T^{\prime}=B \backslash T=\left\{j_{k}\right\}_{k=1}^{n}: \epsilon_{j_{k}}=-$. Different solutions will be parametrized by the sets $\gamma_{1}, \cdots, \gamma_{n-1}$ which will be specified later. The solutions look as follows

$$
\begin{aligned}
& f_{\gamma_{1}, \cdots, \gamma_{n-1}}\left(\lambda_{1}, \cdots, \lambda_{2 n}\right)^{\epsilon_{1}, \cdots, \epsilon_{2 n}}=\prod_{i<j}\left(\lambda_{i}-\lambda_{j}\right)^{\frac{1}{4}} \\
& \times \prod_{i \in T, j \in T^{\prime}}\left(\lambda_{i}-\lambda_{j}\right)^{-1} \operatorname{det}\left\|\int_{\gamma_{i}} \zeta_{j}\left(\tau|T| T^{\prime}\right) d \tau\right\|_{g \times g}
\end{aligned}
$$

$\zeta_{j}$ are the following differentials on the hyper-elliptic surface (HES) $w^{2}=P(\tau) \equiv$ $\prod\left(\tau-\lambda_{i}\right)$ :

$$
\begin{aligned}
& \zeta_{j}\left(\tau|T| T^{\prime}\right)=\frac{Q_{j}\left(\tau|T| T^{\prime}\right)}{\sqrt{P(\tau)}}, \\
& Q_{j}(\tau)=\left\{\prod_{S}\left(\tau-\lambda_{l}\right)\left[\frac{d}{d \tau} \frac{\prod_{S^{\prime}}\left(\tau-\lambda_{l}\right)}{\tau^{n-j}}\right]_{0}+\prod_{S^{\prime}}\left(\tau-\lambda_{l}\right)\left[\frac{d}{d \tau} \frac{\prod_{S}\left(\tau-\lambda_{l}\right)}{\tau^{n-j}}\right]_{0}\right\}
\end{aligned}
$$

where []$_{0}$ means that only the polynomial part of the expression in brackets is taken. The differentials $\zeta_{j}$ are of the second kind: they have singularities at $\infty^{ \pm}$, 
but their residues at the infinities are equal to zero. It should be mentioned that the singular part of the differential $\zeta_{j}$ is independent of the partition of $\Lambda$ :

$$
\zeta_{j}\left(\tau|T| T^{\prime}\right)-\zeta_{j}\left(\tau\left|T_{1}\right| T_{1}^{\prime}\right)=\text { of the first kind }
$$

First kind differentials in our case are of the type: $\sigma_{j}=\tau^{j-1} / \sqrt{P(\tau)}, 1 \leq j \leq n-1$. The contours $\gamma_{1}, \cdots, \gamma_{n-1}$ are arbitrary cycles on the HES ( notice that its genus $g$ equals $n-1)$.

It can be shown that the asymptotics of the form factor $F$ corresponds to the following special choice of $\gamma_{1}, \cdots, \gamma_{n-1}$ : they are taken as canonical $a$-cycles $a_{1}, \cdots, a_{g}$ where the cycle $a_{i}$ surrounds the cut between $\lambda_{2 i-1}$ and $\lambda_{2 i}, i=1, \cdots, n-1$ (we suppose $\lambda$ 's to be ordered: $\lambda_{1}<\lambda_{2}<\cdots<\lambda_{2 n}$ ).

Now, let us turn to the problem of braiding. The equations (5.3) are invariant under the permutation $\lambda_{i} \leftrightarrow \lambda_{j}$ and simultaneous permutation of the associated spaces. Let us denote the operation of the analytical continuation $\lambda_{i} \leftrightarrow \lambda_{i+1}$ and permutation of corresponding spaces by $B_{i, i+1}$. Then we are supposed to have a formula of the type:

$$
\begin{aligned}
& B_{i, i+1} f_{\gamma_{1}, \cdots, \gamma_{n-1}}\left(\lambda_{1}, \cdots, \lambda_{2 n}\right)= \\
& =\sum_{\gamma_{1}^{\prime}, \cdots, \gamma_{n-1}^{\prime}} C_{\gamma_{1}, \cdots, \gamma_{n-1}}^{\gamma_{1}^{\prime}, \cdots, \gamma_{\gamma_{1}}^{\prime}, \cdots, \gamma_{n-1}^{\prime}}\left(\lambda_{1}, \cdots, \lambda_{2 n}\right)
\end{aligned}
$$

where $C$ are some constants. There is one interpretation of the braiding following from the formula (3.3) : $\lambda_{i} \leftrightarrow \lambda_{i+1}$ corresponds to certain element of the modular group $S p(2 g, Z)$ of the HES. Clearly under the braiding the determinants (3.3) transform under $g$-th exterior power of the vector representation of the group. It can be shown that this interpretation implies that the solution $f$ corresponding to $\gamma_{1}, \cdots, \gamma_{g}=a_{1}, \cdots, a_{g}$ mixes by braiding only with those solutions which correspond to other choices of half-basis of homologies ( $g$ contours on the surface with zero intersection numbers). This is the main part of solutions of $\mathrm{KZ}$ defined above. There is the same number of elements in the main part as the number of independent half-bases, i. e. $C_{2 g+2}^{g+1}-C_{2 g+2}^{g}$ of them. On the other hand it is well known nowadays that the braiding is described by the finite-dimensional quantum group $S L(2)_{q}$ [15], in our case $q=-1$. The number of solution coincides with the the multiplicity of the one-dimensional representation in the tensor product of $2 n$ twodimensional representations ot $S L(2)_{q}$ which is in generic situation $C_{2 n}^{n}-C_{2 n}^{n-1}$ (the same as above since $g=n-1$ ). So, it is natural to suppose that the main part of solutions for the case $q=-1$ allows continuation to other $q$ while the rest of solutions is special for $q=-1$. In what follows we shall need only the main part. There is a beautiful way of rewriting the formulae (3.3) for the case of the main part of solutions in terms of $\theta$-functions. We shall consider that in the next section.

Now let us turn to the problem of physical interpretation of the classical limit in question. We have to understand what does the limit $D(Y) \rightarrow \widehat{s l}(2)$ mean. In that limit, in particular, the antipode-square automorphism of $D(Y)$ turns into differentiation for $\widehat{s l}(2)$ :

$$
s^{2} \rightarrow 1+h D
$$

We do not denote $D$ by familiar notation $\left(L_{-1}\right)$ to avoid a confusion: $D$ is not a derivative in the space-time. There is a strange thing about this limit. The 
antipode-square $\left(s^{2}\right)$ is identified with the rotation of the space-time in the theory [6], for two-dimensional representations (particles) it corresponds to the shift of rapidity by $2 \pi i$. So, the operation, $s^{2}$ corresponds to, in the space-time of QFT model is essentially finite being of topological character, hence the consideration of its infinitesimal limit (3.5) does not look very reasonable. However we do will to consider the limit because from mathematical point of view the limits of certain objects (e.g. form factors) do make sense. Thinking more of this situation one comes upon an idea that in the limit in question the very notion of the space-time of the QFT model should be lost. Form factors are the objects referring to one point in the space-time, so their limits in this strange situation might make sense because the notion of one point might remain (it simply appears to be isolated from the rest of the world). Certainly form factors also do not survive in full meaning. First, we deal not with a real limit, but with asymptotical equivalence. Second, the difference disappears between two-dimensional representation and dual to it which contained for $D(Y)$ essential crossing shift, and the two-dimensional representation can not be considered as those corresponding to particles. Thus, the only reasonable guess we can make is that the QFT in the limit splits into a family of systems with finite degrees of freedom, the limits of form factors being somehow connected with these systems. The situation is difficult also because we do not expect any connection between these finite-dimensional system and QFT on Hamiltonian level.

Let us consider the situation in opposite direction. Suppose we have certain family of classical systems every of which has a finite number of degrees of freedom. These systems should be unified through the fact of possessing the symmetry under $\widehat{s l}(2)$. Now we perform a quantization of these systems which essentially leads only to the quantization of $\widehat{s l}(2)(\widehat{s l}(2) \rightarrow D(Y))$. It should be mentioned that there is a jump in quantization of $\widehat{s l}(2)$ : as far as we got a small $h$ in $D(Y)$ it can be immediately rescaled to finite one. So, the quantization provides a finite operation $\left(s^{2}\right)$. Now we introduce a space-time, and identify this operation with the rotation of this space-time. The coordinates are introduced as those respecting this interpretation. As soon as it is done we get the notion of particle, and one space-time point we started with appears to be able to interact with others through exchange of particles. This interpretation seems to be reasonable. In particular it solves the problem of disagreement between local conservation laws in classical and quantum integrable asymptotically free models. In a context close to the present consideration this problem was discussed in [6].

So, now we have to explain what classical systems with finite number of degrees of freedom and $\widehat{s l}(2)$-invariance we have in mind. The answer is implied by the structure of formulae of the solutions of KZ on level zero. They are connected with HES. So, it is natural to suppose that the classical systems in question are stationary finite-gap solutions [16-17] of classical soliton equation with $\widehat{s l}(2)$ symmetry. These systems will be described in Section 5. But before doing that we have to explain the connection between the solutions of $\mathrm{KZ}$ on level zero and Riemann $\theta$-functions. 


\section{KZ equations on level zero and Riemann theta-functions.}

In this section we shall derive a formula which express the solutions of KZ equations on level zero in terms of Riemann $\theta$-functions. Let us first fix the notations and introduce necessary definitions.

We consider the HES $\Sigma$ of genus $g$ with $2 g+2$ real branching points ordered as follows: $\lambda_{1}<\cdots<\lambda_{2 g+2}$. Let us put the cuts between the points $\lambda_{2 i-1}, \lambda_{2 i}$ for $i=1, \cdots, g+1$. The $a$-cycles on the surface are taken in canonical way: the cycle $a_{i}$ surrounds the cut $\lambda_{2 i-1}, \lambda_{2 i}$, for $i=1, \cdots, g$. The $b$-cycle $b_{i}(i=1, \cdots, g)$ starts from one bank of the cut $\lambda_{2 i-1}, \lambda_{2 i}$, reaches the cut $\lambda_{2 g+1}, \lambda_{2 g+2}$ by one sheet, and then returns th the other bank of $\lambda_{2 i-1}, \lambda_{2 i}$ by another sheet. There are $g$ nonsingular differentials on the HES: $\sigma_{j}=\tau^{j-1} / \sqrt{P(\tau)}$. The normalized first kind differentials $\omega_{j}$ are linear combinations of $\sigma$ 's satisfying the condition:

$$
\int_{a_{i}} \omega_{j}=\delta_{i, j}
$$

The martix of periods $\Omega$ is defined as

$$
\Omega_{i j}=\int_{b_{i}} \omega_{j}
$$

The period matrix is symmetric due to Riemann bilinear identity.

The second kind differentials possess singularities, but their residues at the singular points vanish. There is one type of the second kind differentials of particular importance. The differentials of this type are obtained by erasing the dependence on one argument of the two-differentials $\omega^{2}(x, y)$ defined on $\Sigma \times \Sigma$. The differential $\omega^{2}(x, y)$ possesses the only singularity at the diagonal being normalized as

$$
\omega^{2}(x, y)=\frac{1}{(x-y)^{2}} d x d y, \quad x \sim y
$$

Being considered as a differential in one variable ( say $x$ ) it satisfy the normalization condition:

$$
\int_{a_{i}} \omega^{2}=0, \quad \forall i
$$

Finally, the differential $\omega^{2}$ is symmetric: $\omega^{2}(x, y)=\omega^{2}(y, x)$.

Riemann $\theta$-function is defined as follows:

$$
\theta(z \mid \Omega)=\sum_{m \in Z^{g}} \exp \left\{\pi i m^{t} \Omega m+2 \pi i z^{t} m\right\}
$$

where $z \in C^{g}$. The periodicity property says

$$
\theta\left(z+\lambda^{\prime}+\Omega \lambda^{\prime \prime} \mid \Omega\right)=\exp \left\{-\pi i \lambda^{\prime \prime}{ }^{t} \Omega \lambda^{\prime \prime}-2 \pi i \lambda^{\prime \prime} z\right\} \theta(z \mid \Omega), \quad \lambda^{\prime}, \lambda^{\prime \prime} \in Z^{g}
$$

The $\theta$-function with characteristics is defined by:

$$
\theta[\eta](z \mid \Omega)=\exp \left\{\pi i \eta^{\prime \prime}{ }^{t} \Omega \eta^{\prime \prime}+2 \pi i\left(z+\eta^{\prime}\right)^{t} \eta^{\prime \prime}\right\} \theta\left(z+\eta^{\prime}+\Omega \eta^{\prime \prime} \mid \Omega\right)
$$

where $\eta=\left(\eta^{\prime}, \eta^{\prime \prime}\right)$ is a characteristic: the vectors $\eta^{\prime}, \eta^{\prime \prime}$ belong to $R^{g}$ ( $R$ is the field of rational numbers). We will be interested in the case when $\eta$ is a half- period characteristics which means that $\eta^{\prime}, \eta^{\prime \prime} \in 1 / 2 Z^{g}$. 
The definition of $\theta$-function refers to the particular choice of $a$ and $b$ cycles. However, the $\theta$-functions defined with respect to different choices of the homology bases are connected due to the modular property of $\theta$-function. Suppose that we have two homology bases connected via a transformation from $\Gamma_{1,2} \in S p(2 g, Z)$ :

$$
\left(\begin{array}{l}
A^{\prime} \\
B^{\prime}
\end{array}\right)=\left(\begin{array}{ll}
d & c \\
b & a
\end{array}\right)\left(\begin{array}{l}
A \\
B
\end{array}\right), \quad\left(\begin{array}{ll}
d & c \\
b & a
\end{array}\right) \in S p(2 g, Z), \begin{aligned}
& \operatorname{diag} c d^{t} \\
& \operatorname{diag} a b^{t}
\end{aligned} \equiv 0(\bmod 2)
$$

"diag" means the vector composed of the diagonal entries of the matrix. The transformation law for $\theta$-functions says that [19]:

$$
\theta[\xi]\left(z^{\prime} \mid \Omega^{\prime}\right)=\gamma(\operatorname{det}(M))^{1 / 2} \exp \left\{\pi i \sum_{i<j} z_{i} z_{j} \frac{\partial \log (\operatorname{det} M)}{\partial \Omega_{i, j}}\right\} \theta[\eta](z \mid \Omega)
$$

where $M=(c \Omega+d), z=M^{t} z^{\prime}, \Omega^{\prime}=(a \Omega+b)(c \Omega+d)^{-1}$, the characteristics changes as follows

$$
\left(\begin{array}{c}
\xi^{\prime} \\
\xi^{\prime \prime}
\end{array}\right)=\left(\begin{array}{cc}
a & -c \\
-b & d
\end{array}\right)\left(\begin{array}{c}
\eta^{\prime} \\
\eta^{\prime \prime}
\end{array}\right)
$$

$\gamma$ is irrelevant for our goals 8 -th root of unity.

Let us denote the set $\{1,2, \cdots, 2 g+2\}$ counting the branching points of $\Sigma$ by $B$. The most significant property of HES is that they allow one-to-one correspondence between half-periods and the subsets of $B$ with even number of elements (mod identification $T \sim B \backslash T)$ [19]. The correspondence is achieved as follows. Consider the subset $T(\# T=2 m)$ and divide it into two subsets $T_{1}, T_{2}$ such that $\# T_{1}=$ $\# T_{2}=m$. Now let $T_{1}=\left\{i_{1}, \cdots, i_{m}\right\}, T_{2}=\left\{j_{1}, \cdots, j_{m}\right\}$ and associate to $T$ the half-period characteristic $\tilde{\eta}_{T}$ such that

$$
\tilde{\eta}_{T}^{\prime}+\Omega \tilde{\eta}_{T}^{\prime \prime}=\sum_{k} \int_{\lambda_{i_{k}}}^{\lambda_{j_{k}}} \omega
$$

where $\omega$ is considered as vector composed of first kind differentials. It can be easily shown that the the ambiguity in dividing of $T$ into subsets and enumerating these subsets changes $\tilde{\eta}_{T}$ by full period which can be ignored. It is convenient to measure the characteristics relative to Riemann characteristic $\delta$ which is defined as

$$
\delta=\left\{\begin{array}{cc}
\tilde{\eta}_{U}, & \text { if } g \equiv 1(\bmod 2) \\
\tilde{\eta}_{\{U \backslash 1\}}, & \text { if } g \equiv 0(\bmod 2)
\end{array}\right.
$$

where $U=\{1,3,5, \cdots\}$. The characteristic $\eta_{T}$ corresponding to the subset $T$ such that $\# T \equiv g+1(\bmod 2)$ is defined by

$$
\eta_{T}=\tilde{\eta}_{T \circ U}
$$

where $T \circ U=(T \cup U) \backslash(T \cap U)$.

Our nearest goal is to prove the following

Proposition. Consider the solution of $\mathrm{KZ}$ on level zero which corresponds to $\gamma_{1}, \cdots, \gamma_{g}=a_{1}, \cdots, a_{g}$. The components of this solution are denoted by 
$f\left(\lambda_{1}, \cdots, \lambda_{2 g+2}\right)^{\epsilon_{1}, \cdots, \epsilon_{2 g+2}}$. To every multiindex $\epsilon_{1}, \cdots, \epsilon_{2 n}$ a partition of $B$ corresponds such that $B=T \cup T^{\prime}, \quad i \in T: \epsilon_{i}=+, i \in T^{\prime}: \epsilon_{i}=-$. Denote the elements of $T$ by $i_{p}, p=1, \cdots, g+1$. The set $T$ is associated with $\theta$ - characteristic $\eta_{T}$. The following relation holds:

$$
f\left(\lambda_{1}, \cdots, \lambda_{2 g+2}\right)^{\epsilon_{1}, \cdots, \epsilon_{2 g+2}}=C^{-3} \theta\left[\eta_{T}\right](0)^{4} \operatorname{det}\left\|\partial_{p} \partial_{q} \log \theta\left[\eta_{T}\right](0)\right\|_{g \times g}
$$

where $C$ is the constant: $C=\prod_{i<j}\left(\lambda_{i}-\lambda_{j}\right)^{\frac{1}{4}} \Delta, \Delta$ is the following important in future determinant:

$$
\Delta=\operatorname{det}\left\|\int_{a_{i}} \sigma_{j}\right\|_{g \times g}
$$

The notations are used:

$$
\left.\partial_{i} F(\cdots, 0, \cdots) \equiv \frac{\partial}{\partial z_{i}} F\left(\cdots, z_{i}, \cdots\right)\right|_{z_{i}=0}
$$

To prove the proposition we have to explain certain facts concerning $\theta$-functions on HES.

Tomae formulae says that $\theta\left[\eta_{T}\right](0)=0$ if $\# T \neq g+1$, and if $\# T=g+1$ then

$$
\theta\left[\eta_{T}\right](0)^{2}=\prod_{i<j, i, j \in T}\left(\lambda_{i}-\lambda_{j}\right)^{\frac{1}{2}} \prod_{i<j, i, j \in B \backslash T}\left(\lambda_{i}-\lambda_{j}\right)^{\frac{1}{2}} \Delta
$$

The following remarkable relation between the differentials $\omega^{2}$ the derivatives of $\theta$-function at $z=0$ ( $\theta$-constants) takes place. If $x, y, \eta$ are such that $\theta[\eta]\left(\int_{x}^{y} \omega\right)=0$ then [20]:

$$
\omega^{2}(x, y)=-\sum_{i, j} \partial_{i} \partial_{j} \log \theta[\eta](0) \omega_{i}(x) \omega_{j}(y)
$$

To proceed further we need explicit formulae for certain differentials. The normalized first kind differential $\omega_{i}$ is given by $g \times g$ determinant:

$$
\omega_{i}(x)=(-1)^{i} \Delta^{-1} \operatorname{det}\left(\begin{array}{cccccccc}
\sigma_{1}(x), & \int_{a_{1}} \sigma_{1} & \cdots & \int_{a_{i-1}} \sigma_{1}, & \int_{a_{i+1}} \sigma_{1}, & \cdots & \int_{a_{g}} \sigma_{1} \\
\vdots & \vdots & \vdots & \vdots & \vdots & \vdots & \vdots \\
\sigma_{g}(x), & \int_{a_{1}} \sigma_{g} & \cdots & \int_{a_{i-1}} \sigma_{g}, & \int_{a_{i+1}} \sigma_{g}, & \cdots & \int_{a_{g}} \sigma_{g}
\end{array}\right)
$$

Let us consider now the differential $\omega^{2}(x, y)$ as function of $x$ specifying $y$ to one of the branching points: $y=\lambda_{i}$. We take $\xi=\sqrt{\left(\tau-\lambda_{i}\right)}$ as local parameter in vicinity of $\lambda_{i}$. The differential $\omega^{2}\left(x, \lambda_{i}\right)$ is second kind differentil with zero $a$-periods and fixed singularity: $\omega^{2}\left(x, \lambda_{i}\right) \sim \xi^{-2}, x \sim \lambda_{i}$. It can be presented as $(g+1) \times(g+1)$ determinant:

$$
\omega^{2}\left(x, \lambda_{i}\right)=\Delta^{-1}\left(P^{\prime}\left(\lambda_{i}\right)\right)^{\frac{1}{2}} \operatorname{det}\left(\begin{array}{ccccc}
\sigma_{1}(x), & \int_{a_{1}} \sigma_{1} & \cdots & \cdots & \int_{a_{g}} \sigma_{1} \\
\vdots & \vdots & \vdots & \vdots & \vdots \\
\sigma_{g}(x), & \int_{a_{1}} \sigma_{g} & \cdots & \cdots & \int_{a_{g}} \sigma_{g} \\
\rho_{i}(x), & \int_{a_{1}} \rho_{i} & \cdots & \cdots & \int_{a_{g}} \rho_{i} \\
13 & & & &
\end{array}\right)
$$


where $\rho_{i}(\tau)=\frac{1}{\left(\tau-\lambda_{i}\right) \sqrt{P(\tau)}}, \quad P^{\prime}\left(\lambda_{i}\right) \equiv \prod_{j}^{\prime}\left(\lambda_{i}-\lambda_{j}\right)$. From this formula one derive the following representation for $\omega^{2}\left(x, \lambda_{i}\right)$ when $x=\lambda_{j}$ in terms of $g \times g$ determinant:

$$
\omega^{2}\left(\lambda_{j}, \lambda_{i}\right)=\Delta^{-1}\left(\frac{P^{\prime}\left(\lambda_{i}\right)}{P^{\prime}\left(\lambda_{j}\right)}\right)^{\frac{1}{2}} \operatorname{det}\left(\begin{array}{cccc}
\int_{a_{1}} \sigma_{2, j} & \cdots & \cdots & \int_{a_{g}} \sigma_{2, g} \\
\vdots & \vdots & \vdots & \vdots \\
\int_{a_{1}} \sigma_{g, j} & \cdots & \cdots & \int_{a_{g}} \sigma_{g, j} \\
\int_{a_{1}} \rho_{i, j} & \cdots & \cdots & \int_{a_{g}} \rho_{i, j}
\end{array}\right)
$$

where $\sigma_{l, j}=\sigma_{l}-\lambda_{j} \sigma_{l-1}, \quad \rho_{i, j}=\rho_{i}+\left(\lambda_{j}-\lambda_{i}\right)^{-1} \sigma_{1}$. Combining (4.6) and (4.5) one arrives at

$$
\sum \partial_{p} \partial_{q} \theta\left[\eta_{T}\right](0) A_{u}^{p} A_{v}^{q} \lambda_{i}^{u} \lambda_{j}^{v}=\Delta^{-1} P^{\prime}\left(\lambda_{i}\right) \operatorname{det}\left(\begin{array}{cccc}
\int_{a_{1}} \sigma_{2, j} & \cdots & \cdots & \int_{a_{g}} \sigma_{2, g} \\
\vdots & \vdots & \vdots & \vdots \\
\int_{a_{1}} \sigma_{g, j} & \cdots & \cdots & \int_{a_{g}} \sigma_{g, j} \\
\int_{a_{1}} \rho_{i, j} & \cdots & \cdots & \int_{a_{g}} \rho_{i, j}
\end{array}\right)
$$

where $i, j \in T$, the matrix $A$ connects normalized first kind differentials with trivial ones:

$$
\omega=A \sigma, \quad A=\left\|\int_{a_{i}} \sigma_{j}\right\|^{-1}
$$

The reason for the equation (4.7) to hold is that adding $\int_{\lambda_{i}}^{\lambda_{j}} \omega$ to $\eta_{T}$ we get odd characteristics corresponding to $T \backslash\{i, j\}$, and $\theta\left[\eta_{T \backslash\{i, j\}}\right](0) \stackrel{\lambda_{i}}{=} 0$.

Let us return to the solution of KZ equations corresponding to $\gamma_{1}, \cdots, \gamma_{g}=$ $a_{1}, \cdots, a_{g}$. It is given by (3.3):

$$
\begin{gathered}
\left.f_{(} \lambda_{1}, \cdots, \lambda_{2 g+2}\right)^{\epsilon_{1}, \cdots, \epsilon_{2 g+2}}=\prod_{i<j}\left(\lambda_{i}-\lambda_{j}\right)^{\frac{1}{4}} \\
\prod_{i \in T, j \in T^{\prime}}\left(\lambda_{i}-\lambda_{j}\right)^{-1} \operatorname{det}\left\|\int_{a_{i}} \zeta_{j}\left(\tau|T| T^{\prime}\right) d \tau\right\|_{g \times g}
\end{gathered}
$$

where $T, T^{\prime}$ are associated to $\epsilon_{1}, \cdots, \epsilon_{g}$ in a usual way. Let us take $g$ elements from $T$ (say $i_{p}, p=1, \cdots, g$ ) and multiply the determinant from (4.9) by Vandermond composed of corresponding $\lambda$ 's the result being

$$
\operatorname{det}|| F_{p, q} \|_{g \times g}, \quad F_{p, q}=\int_{a_{p}} \sum_{m=1}^{g} \zeta_{m}{\lambda_{i_{q}}}^{g-m}
$$

The following two differentials are equivalent (differ by a total derivative):

$$
\sum_{m=1}^{g} \zeta_{m} \lambda_{i_{q}}{ }^{g-m} \sim \mu_{q} \equiv \prod_{j \in B \backslash T}\left(\lambda_{i_{q}}-\lambda_{j}\right) \frac{\prod_{i \in T, i \neq i_{q}}\left(\tau-\lambda_{i}\right)}{\left(\tau-\lambda_{i_{q}}\right) \sqrt{P(\tau)}}
$$

Hence the determinant in (4.9) can be replaced by

$$
\frac{1}{\prod_{i<i^{\prime}, i, i^{\prime} \in T \backslash i_{g+1}}\left(\lambda_{i}-\lambda_{i^{\prime}}\right)} \operatorname{det}(C), \quad C_{p q}=\int_{a_{p}} \mu_{q}
$$


Let us find the matrix $X$ which satisfies the equation:

$$
A X=C
$$

with $A$ and $C$ given by (4.8),(4.10). Kramer's rule tells that

$$
X_{p, q}=(-1)^{p} \Delta^{-1} \operatorname{det}\left(\begin{array}{ccccc}
\int_{a_{1}} \sigma_{1} & \cdots & \cdots & \cdots & \int_{a_{g}} \sigma_{1} \\
\vdots & \vdots & \vdots & \vdots & \vdots \\
\int_{a_{1}} \sigma_{p-1} & \cdots & \cdots & \cdots & \int_{a_{g}} \sigma_{p-1} \\
\int_{a_{1}} \sigma_{p+1} & \cdots & \cdots & \cdots & \int_{a_{g}} \sigma_{p+1} \\
\vdots & \vdots & \vdots & \vdots & \vdots \\
\int_{a_{1}} \sigma_{g} & \cdots & \cdots & \cdots & \int_{a_{g}} \sigma_{g} \\
\int_{a_{1}} \mu_{q} & \cdots & \cdots & \cdots & \int_{a_{g}} \mu_{q}
\end{array}\right)
$$

Let us now consider the determinant of the matrix $X$. Multiplying it by Vandermond composed of $\lambda_{i_{k}}, k=1, \cdots, g$ one gets

$$
\operatorname{det}(X)=\frac{1}{\prod_{i<i^{\prime}, i, i^{\prime} \in T \backslash i_{g+1}}\left(\lambda_{i}-\lambda_{i^{\prime}}\right)} \operatorname{det}(\tilde{X})
$$

where

$$
\tilde{X}_{p, q}=\Delta^{-1} \operatorname{det}\left(\begin{array}{ccccc}
\int_{a_{1}} \sigma_{2, p} & \cdots & \cdots & \cdots & \int_{a_{g}} \sigma_{2, p} \\
\vdots & \vdots & \vdots & \vdots & \vdots \\
\int_{a_{1}} \sigma_{g, p} & \cdots & \cdots & \cdots & \int_{a_{g}} \sigma_{g, p} \\
\int_{a_{1}} \mu_{q} & \cdots & \cdots & \cdots & \int_{a_{g}} \mu_{q}
\end{array}\right)
$$

It is easy to show that $\mu_{q}$ in $(4.12)$ can be replaced by $P^{\prime}\left(\lambda_{i_{q}}\right) \rho_{i_{p}, i_{q}}$. Combining that with (4.7) and taking into account that $\operatorname{det}(A)=\Delta^{-1}$ one gets:

$$
\operatorname{det}(X)=\prod_{i<i^{\prime}, i, i^{\prime} \in T \backslash i_{g+1}}\left(\lambda_{i}-\lambda_{i^{\prime}}\right) \operatorname{det}\left\|\partial_{p} \partial_{q} \log \theta\left[\eta_{T}\right](0)\right\|_{g \times g}
$$

Calculating determinants of RHS and LHS of formulae (4.11) and having in mind (4.8) we obtain:

$$
\operatorname{det}\left\|\int_{a_{i}} \zeta_{j}\right\|=\Delta \operatorname{det}\left\|\partial_{p} \partial_{q} \log \theta\left[\eta_{T}\right](0)\right\|
$$

Together with Tomae formulae it provides the representation for the solutions of $\mathrm{KZ}$ on level 0:

$$
f\left(\lambda_{1}, \cdots, \lambda_{2 g+2}\right)^{\epsilon_{1}, \cdots, \epsilon_{2 g+2}}=C^{-3} \theta\left[\eta_{T}\right](0)^{4} \operatorname{det}\left\|\partial_{p} \partial_{q} \log \theta\left[\eta_{T}\right](0)\right\|_{g \times g}
$$

where $T$ is related to $\epsilon_{1}, \cdots, \epsilon_{2 g+2}$ as explained above, $C=\prod_{i<j}\left(\lambda_{i}-\lambda_{j}\right)^{\frac{1}{4}} \Delta$. That proves the proposition above.

The formula (4.3) has very beautiful meaning. It relates the solution of KZ equations which are differential equation with respect to moduli of HES (the positions 
of branching points) in terms of derivatives on the Jacobian. It would be nice to prove directly that (4.3) satisfies $\mathrm{KZ}$ using the heat equation for the $\theta$-function.

Now suppose that we took a solution corresponding to other choice of half basis: $\gamma_{1}, \cdots, \gamma_{g}=a_{1}^{\prime}, \cdots, a_{g}^{\prime}$. Denote this solution by $f_{A^{\prime}}\left(\lambda_{1}, \cdots, \lambda_{2 g+2}\right)$. Clearly, all the reasonings above are applicable to $f_{A^{\prime}}$ the difference being that the final formula has to contain $\theta$-functions defined with respect to the half-basis $A^{\prime}$. In order to rewrite the answer in terms of canonical $\theta$-functions (those corresponding to $A$ ) we have to use the formula (4.1). After simple computations one gets:

$$
\begin{aligned}
& f_{A^{\prime}}\left(\lambda_{1}, \cdots, \lambda_{2 g+2}\right)^{\epsilon_{1}, \cdots, \epsilon_{2 g+2}}= \\
& \operatorname{det}(M) C^{-3} \theta\left[\eta_{T}\right](0)^{4} \operatorname{det}\left\|\partial_{p} \partial_{q} \log \theta\left[\eta_{T}\right](0)+\pi i \frac{\partial \log (\operatorname{det} M)}{\partial \Omega_{i, j}}\right\|
\end{aligned}
$$

where

$$
\left(\begin{array}{l}
A^{\prime} \\
B^{\prime}
\end{array}\right)=\left(\begin{array}{cc}
d & c \\
b & a
\end{array}\right)\left(\begin{array}{l}
A \\
B
\end{array}\right), \quad\left(\begin{array}{cc}
d & c \\
b & a
\end{array}\right) \in \Gamma_{1,2}
$$

The matrix $M$ is defined as $M=(c \Omega+d)$.

In Section 6 we shall need another representation for the solutions of KZ in terms of $\theta$-functions. The author was unable to prove this representation completely, so we shall formulate it as conjecture and present reasonings in favour of it.

Conjecture 1. For every half-basis $A^{\prime}$ a polynomial $Q_{A^{\prime}}$ in $\partial_{i}$ of total degree $2 g$ exists such that

$$
f_{A^{\prime}}\left(\lambda_{1}, \cdots, \lambda_{2 g+2}\right)^{\epsilon_{1}, \cdots, \epsilon_{2 g+2}}=C^{-3} \theta\left[\eta_{T}\right](0)^{2} Q_{A^{\prime}} \theta\left[\eta_{T}\right](0)^{2}
$$

where the connection between $\epsilon_{1}, \cdots, \epsilon_{2 g+2}$ and $T$ ia as usual, the coefficients of $Q_{A^{\prime}}$ might be complicated, but they do not depend on $T$.

Let us explain why we assume the representation to exist. To this end we have to understand what kind of constants depending on $T$ is a candidate for being presentable in the form $\theta\left[\eta_{T}\right](0)^{2} Q \theta\left[\eta_{T}\right](0)^{2}$ for some polynomial in $\partial_{i}$ and $T$ corresponding to even non-singular characteristics $(\# T=g+1)$. Riemann $\theta$-functions on HES satisfy many relations which follow from the combination of Riemann relations with the peculiar properties of HES. In particular, the following relations hold for $\theta[\eta](z)$ ( $\eta$ is even, nonsingular, i.e. such that $\theta[\eta](0) \neq 0)[19]$ :

$$
\sum_{S \subset B, \# S=g+1,1 \in S}(-1)^{S \cap(U \circ T)} \theta\left[\eta_{S}\right](z)^{2} \theta\left[\eta_{S}\right](0)^{2}=0
$$

where $T$ is arbitrary subset of $B$ satisfying the requirements: $\# T \equiv g+1(\bmod 2)$, $\# T \neq g+1,1 \in T$. We put the requirement $1 \in S$ in order to avoid the summation over $B \backslash S$. So, if the set of constants is presentable in the form $Q \theta\left[\eta_{S}\right](0)^{2}$ then they should satisfy this system of relations being placed instead of $\theta\left[\eta_{S}\right](z)^{2}$. The opposite should be also true: if a set of constants enumerated by $S$ satisfies the system (4.15) then they can be presented in the form $Q \theta\left[\eta_{S}\right](0)^{2}$. In elliptic case $(g=1)$ there is only one set $T$ satisfying the requirements above: $T=B$. For this set the relation (4.15) turns into

$$
\theta_{0,0}(0)^{2} \theta_{0,0}(z)^{2}=\theta_{1,0}(0)^{2} \theta_{1,0}(z)^{2}+\theta_{0,1}(0)^{2} \theta_{0,1}(z)^{2}
$$


in usual notation for the elliptic $\theta$-functions with characteristics [19]:

$$
\theta_{1,0}(z)=\theta\left[\eta_{\{1,2\}}\right](z), \theta_{0,0}(z)=\theta\left[\eta_{\{1,3\}}\right](z), \theta_{0,1}(z)=\theta\left[\eta_{\{1,4\}}\right](z) .
$$

Let us prove that the solutions of KZ for arbitrary choice of $A^{\prime}$ satisfy the relations (4.15). First, let us check that $\theta\left[\eta_{S}\right](0)^{2}$ given by Tomae formulae satisfy the requirements. Substitute $\theta\left[\eta_{S}\right](0)^{2}$ given by (4.4) into (4.15) instead of both $\theta\left[\eta_{S}\right](0)^{2}$ and $\theta\left[\eta_{S}\right](z)^{2}$ and divide the relation by $\Delta^{2} \prod_{i<j}\left(\lambda_{i}-\lambda_{j}\right)$ the result being

$$
\sum_{S \subset B, \# S=g+1,1 \in S}(-1)^{S \cap(U \circ T)} \frac{1}{\prod_{i \in S, j \in B \backslash S}\left(\lambda_{i}-\lambda_{j}\right)}=0
$$

These identities are proven by consideration of residues at $\lambda_{i}=\lambda_{j}, \forall i, j$.

Now let us present the solution of KZ (4.9) as multidimensional integral:

$$
\begin{aligned}
& f_{A^{\prime}}\left(\lambda_{1}, \cdots, \lambda_{2 g+2}\right)^{\epsilon_{1}, \cdots, \epsilon_{2 g+2}}=\prod_{i<j}\left(\lambda_{i}-\lambda_{j}\right)^{\frac{1}{4}} \\
& \times \prod_{i \in S, j \in B \backslash S}\left(\lambda_{i}-\lambda_{j}\right)^{-1} \int_{a_{1}^{\prime}} d \tau_{1} \frac{1}{\sqrt{P\left(\tau_{1}\right)}} \cdots \int_{a_{g}^{\prime}} d \tau_{g} \frac{1}{\sqrt{P\left(\tau_{g}\right)}} \operatorname{det}|| Q_{i}\left(\tau_{j}|S| S^{\prime}\right) \|_{g \times g}
\end{aligned}
$$

where $Q_{i}$ are given by (3.4), $S$ is related to $\epsilon_{1}, \cdots, \epsilon_{2 g+2}$ as usual. Let us denote $\operatorname{det}|| Q_{i}\left(\tau_{j}\right) \|_{g \times g}$ by $X_{g}\left(\tau_{1}, \cdots, \tau_{g}|S| S^{\prime}\right)$ indicating the dependence on genus $g$ and subsets $S, S^{\prime}$ (recall that $S^{\prime}=B \backslash S$ ). We shall prove that the solutions of $\mathrm{KZ}$ satisfy (4.15) being substituted as $\theta\left[\eta_{S}\right](0)^{2} \theta\left[\eta_{S}\right](z)^{2}$ if we prove that $\prod_{i \in S, j \in S^{\prime}}\left(\lambda_{i}-\right.$ $\left.\lambda_{j}\right)^{-1} X_{g}\left(\tau_{1}, \cdots, \tau_{g}|S| S^{\prime}\right)$ satisfy them. So, we have to prove that

$$
\sum_{S \subset B, \# S=g+1,1 \in S}(-1)^{S \cap(U \circ T)} \prod_{i \in S, j \in S^{\prime}}\left(\lambda_{i}-\lambda_{j}\right)^{-1} X_{g}\left(\tau_{1}, \cdots, \tau_{g}|S| S^{\prime}\right)=0
$$

In fact the proof of the identity does not differ much from the proof of (4.16). Again it is sufficient to check the cancellation of the residues at $\lambda_{i}=\lambda_{j}$ because $X_{g}\left(\tau_{1}, \cdots, \tau_{g}|S| S^{\prime}\right)$ is of degree $g$ with respect to any $\lambda$. Suppose $l \in S, m \in S^{\prime}$ then the polynomial $X_{g}\left(\tau_{1}, \cdots, \tau_{g}|S| S^{\prime}\right)$ satisfies the following recurrent relation (classical version of the relations from [11]):

$$
\begin{aligned}
& \left.X_{g}\left(\tau_{1}, \cdots, \tau_{g}|S| S^{\prime}\right)\right|_{\lambda_{l}=\lambda_{m}}=\prod_{k=1}^{g}\left(\tau_{k}-\lambda_{l}\right) \sum_{k=1}^{g}(-1)^{k}\left\{\frac{\partial}{\partial \tau_{k}} \prod_{p \in B \backslash\{l, m\}}\left(\tau_{k}-\lambda_{p}\right)\right\} \\
& \times X_{g-1}\left(\tau_{1}, \cdots, \tau_{k-1}, \tau_{k+1}, \cdots, \tau_{g}|S \backslash l| S^{\prime} \backslash m\right)
\end{aligned}
$$

The point is that the coefficients in the relation are independent of $S$. That provides the possibility of inductive proof of (4.17).

It would be nice to have explicit formulae for the polynomials $Q$ in (4.14). The author has not succeeded to get them. Presumably the formulae can be found from (4.3) using Fay identities [20].

To finish this section let us present explicit formulae for the elliptic case. The canonic way for construction of homology basis is as follows: the cycle $a$ surrounds the cut between $\lambda_{1}, \lambda_{2}$, the cycle $b$ starts from upper bank of this cut, reaches 
the upper bank of the cut $\lambda_{3}, \lambda_{4}$ by one sheet, then moves to another sheet and starting with the lower bank of $\lambda_{3}, \lambda_{4}$ returns to the lower bank $\lambda_{1}, \lambda_{2}$. There are two independent choices of $a$-cycle: $a=a$ or $a^{\prime}=b$ which are connected by the following element from $S p(2, Z)$ :

$$
\left(\begin{array}{cc}
0 & 1 \\
-1 & 0
\end{array}\right)
$$

So, there are two independent solutions to KZ:

$$
\begin{aligned}
& f_{a}\left(\lambda_{1}, \cdots, \lambda_{4}\right)^{\epsilon_{1}, \cdots, \epsilon_{4}}=C^{-3} \theta\left[\eta_{T}\right](0)^{4} \partial^{2} \log \theta\left[\eta_{T}\right](0), \\
& f_{a^{\prime}}\left(\lambda_{1}, \cdots, \lambda_{4}\right)^{\epsilon_{1}, \cdots, \epsilon_{4}}=C^{-3} \theta\left[\eta_{T}\right](0)^{4} K\left[\partial^{2} \log \theta\left[\eta_{T}\right](0)+K^{-1}\right]
\end{aligned}
$$

where $K$ is full elliptic integral: $K=\frac{1}{\pi i} \int_{\lambda_{1}}^{\lambda_{2}} \frac{d \tau}{\sqrt{P(\tau)}}$, the correspondence between $\epsilon_{1}, \cdots, \epsilon_{4}$, $T$ and conventional notations for elliptic $\theta$-functions are as follows:

$$
\begin{aligned}
& \{++--\},\{--++\} \rightarrow\{1,2\} \rightarrow\{1,0\}, \\
& \{+--+\},\{-++-\} \rightarrow\{1,4\} \rightarrow\{0,1\}, \\
& \{+-+-\},\{-+-+\} \rightarrow\{1,3\} \rightarrow\{0,0\}
\end{aligned}
$$

The solution can be also rewritten as (4.14) with

$$
Q_{a}=\partial^{2}, \quad Q_{a^{\prime}}=K \partial^{2}+1
$$

since $\partial \theta[\eta](0)=0$ for even $\eta$. 


\section{Pragmatic view of finite gap integration.}

Consider an integrable equation with infinitely many times $t_{1}, t_{2}, \cdots$. To any time $t_{i}$ the $M$-operator $\mathfrak{M}_{i}$ is attached. The $M$-operators satisfy zero curvature condition:

$$
\frac{\partial}{\partial t_{i}} \mathfrak{M}_{j}-\frac{\partial}{\partial t_{j}} \mathfrak{M}_{i}=\left[\mathfrak{M}_{i}, \mathfrak{M}_{j}\right]
$$

We consider $\widehat{s l}(2)$-invariant case (one can think of nonlinear Shrödinger model for example), so $\mathfrak{M}_{i}$ is traceless $2 \times 2$ matrix depending on spectral parameter $\lambda$ such that

$$
\operatorname{tr}_{i}(\lambda)=0, \mathfrak{M}_{i}(\lambda)=\sum_{k=0}^{i} \lambda^{k} m_{i, k},
$$

also we normalize it by the requirement $m_{i, i}=\sigma^{3}$.

The coefficients of the $M$-operators are dynamical variables. Finite-gap integration [16-18] deals with the situation of stationary solutions which means that there is a time $t_{n}$ on which the dynamical variables do not depend:

$$
\frac{\partial}{\partial t_{n}} \mathfrak{M}_{i}=0, \quad \forall i
$$

That means that the $M$-operator $\mathfrak{M}_{n}$ satisfies the equation:

$$
\frac{\partial}{\partial t_{i}} \mathfrak{M}_{n}=\left[\mathfrak{M}_{i}, \mathfrak{M}_{n}\right], \quad \forall i
$$

hence the determinant of $\mathfrak{M}_{n}$ is an integral of motion with respect to all the times. Certainly this determinant can be presented as follows:

$$
\operatorname{det} \mathfrak{M}_{n}(\lambda)=\prod_{k=1}^{2 n}\left(\lambda-\lambda_{k}\right)
$$

That suggests that the problem in question is closely related to the problem of parametrization of all matrices $M(\lambda)$ (we omit the index $n$ ) which satisfy the following requirements: $M(\lambda)$ is traceless $2 \times 2$ matrix depending on $\lambda$ as polynomial of degree $n$ with fixed senior coefficient (equal $\sigma^{3}$ ) and given determinant $\left(\operatorname{det} M(\lambda)=\prod_{k=1}^{2 n}\left(\lambda-\lambda_{k}\right)\right)$. This problem was considered in the last century (Jacobi, Riemann), the solution can be found for example in Mumford's book [19]. Let us describe it in the terms appropriate for our further goals.

The matrix in question is degenerate at the points $\lambda=\lambda_{j}$, also it is traceless, hence $M\left(\lambda_{j}\right)$ is a Jordan cell:

$$
M\left(\lambda_{j}\right)=\psi_{j} \otimes \bar{\psi}_{j} \quad \bar{\psi}_{j} \equiv \psi_{j}^{t} \sigma^{2}
$$

for some vector $\psi_{j}$. Let us parametrize $M(\lambda)$ by the set $\psi_{j}, j=1, \cdots, 2 n$ (these vectors are not independent as we shall see soon). Construct the interpolation

$$
M^{\prime}(\lambda)=\sum_{k=1}^{2 n} \frac{\prod_{p \neq k}\left(\lambda-\lambda_{p}\right)}{\prod_{p \neq k}\left(\lambda_{k}-\lambda_{p}\right)} M_{k}
$$


Definitely, $M^{\prime}$ is degenerated at the given points with given values, but its degree in $\lambda$ equals $2 n-1$ instead of $n$ required. So, we have to kill $n-1$ senior coefficients of $M^{\prime}$, also we have to take into account that $\lambda^{n}$ is supposed to enter with given coefficient $\sigma^{3}$. After some simple manipulations these requirements lead to the following system of quadratic relations for the components $a_{i}, b_{i}$ of the vectors $\psi_{i}$ :

$$
\begin{aligned}
& \sum_{k \in T} \frac{1}{\prod_{j \in T}^{\prime}\left(\lambda_{k}-\lambda_{j}\right)} a_{k}^{2}=0, \\
& \sum_{k \in T} \frac{1}{\prod_{j \in T}^{\prime}\left(\lambda_{k}-\lambda_{j}\right)} b_{k}^{2}=0, \\
& \sum_{k \in T} \frac{1}{\prod_{j \in T}^{\prime}\left(\lambda_{k}-\lambda_{j}\right)} a_{k} b_{k}=1
\end{aligned}
$$

for any subset $T$ of $n+1$ elements of the set $\{1,2, \cdots, 2 n\}$. This system of equations leaves only $n-1$ independent parameters (which could be, certainly, calculated from the very beginning). The important point is that due to Riemann identities and Tomae formulae the relations allow a parametrization in Riemann $\theta$ functions on the hyperelliptic surface $\tau^{2}=P(\lambda) \equiv \prod_{k=1}^{2 n}\left(\lambda-\lambda_{k}\right)$. The solution to the system (5.2) looks as follows:

$$
a_{j}=C \frac{\theta\left[\eta_{j}\right](r+z)}{\theta[\delta](z) \theta\left[\eta_{j}\right](r)}, b_{j}=C \frac{\theta\left[\eta_{j}\right](r-z)}{\theta[\delta](z) \theta\left[\eta_{j}\right](r)}
$$

where $\eta_{j}$ is the theta-characteristic corresponding to the branching point $\lambda_{j}: \eta_{j}=$ $\tilde{\eta}_{1, j}$ with $\tilde{\eta}_{T}$ defined by (4.2). The variable $z=\left(z_{1}, \cdots, z_{g}\right)$ is the parameter on the Jacobian (genus $g=n-1$ ),

$$
r=g \int_{\lambda_{1}}^{\infty^{+}} \omega,
$$

$\infty^{+}$is one of two infinities on the surface, $\omega$ is the vector composed of first kind differentials, $\delta$ is Riemann constant, finally, $C$ is the same as in (4.3).

We would like to make two comments on the above formulae in order to clarify them from two points of view. First point is the connection with more familiar in the context of finite-gap integration object, namely with Baker-Akhiezer function. The BA function is an eigenvector of $M(\lambda)$ :

$$
M(\lambda) \psi(\lambda)=m(\lambda) \psi(\lambda)
$$

where $m$ is corresponding eigenvalue. Certainly, for generic $\lambda$ there are two solution to this equation which means that the BA function is defined on the surface $\Sigma$. But for $\lambda=\lambda_{i}$ the matrix $M(\lambda)$ is degenerate, it has only one eigenvector corresponding to zero eigenvalue. This eigenvector is exactly our $\psi_{i}$. Thus,

$$
\psi_{i}=\psi\left(\lambda_{i}\right)
$$

The BA-function $\psi(x)\left(x=\lambda^{ \pm}\right.$is a point on the surface) is written in terms of $\theta$ functions through $\theta\left(\int^{x} \omega+\cdots\right),[16-18]$ when $x$ coincides with one of the branching 
points this expression turns into $\theta$-function with corresponding half-period characteristics because the integral of $\omega$ taken between two branching points on hyperelliptic surface is a half-period.

The second point is the direct connection with well known addition theorems for $\theta$-functions in the elliptic case ( $n=2$, there are four branching points). In that case the following identification can be done with usual $\theta$-functions [19]:

$$
\begin{aligned}
& \theta\left[\eta_{1}\right](z)=\theta_{0,0}(z), \theta\left[\eta_{2}\right](z)=\theta_{0,1}(z), \\
& \theta\left[\eta_{3}\right](z)=\theta_{1,1}(z), \theta\left[\eta_{4}\right](z)=\theta_{1,0}(z)
\end{aligned}
$$

Also the following simple variant of Tomae formulae holds:

$$
\begin{aligned}
& \theta_{0,0}(0)^{2}=\left[\left(\lambda_{1}-\lambda_{3}\right)\left(\lambda_{2}-\lambda_{4}\right)\right]^{\frac{1}{2}} K, \theta_{0,1}(0)^{2}=\left[\left(\lambda_{1}-\lambda_{4}\right)\left(\lambda_{2}-\lambda_{3}\right)\right]^{\frac{1}{2}} K \\
& \theta_{1,0}(0)^{2}=\left[\left(\lambda_{1}-\lambda_{2}\right)\left(\lambda_{3}-\lambda_{4}\right)\right]^{\frac{1}{2}} K, \theta_{1,1}(0)^{2}=0
\end{aligned}
$$

where $K$ is the full elliptic integral $K=\frac{1}{\pi i} \int_{a} d \tau / \sqrt{P(\tau)}, a$-cycle surrounds the cut between the points $\lambda_{1}$ and $\lambda_{2}$. The ratios of $\theta$-functions at $r$ are easy to calculate:

$$
\theta\left[\eta_{j}\right](r)^{2}=\text { Const } P^{\prime}\left(\lambda_{j}\right)^{-\frac{1}{2}}, P^{\prime}\left(\lambda_{j}\right) \equiv \prod_{k \neq j}\left(\lambda_{j}-\lambda_{k}\right)
$$

Taking all that into account one realizes that for the parametrization (5.3) considered the equations (5.2) turn, for example for $T=\{1,2,4\}$ into

$$
\begin{aligned}
& \theta_{0,0}(0)^{2} \theta_{0,0}(r+z)^{2}-\theta_{0,1}(0)^{2} \theta_{0,1}(r+z)^{2}-\theta_{1,0}(0)^{2} \theta_{1,0}(r+z)^{2}=0, \\
& \theta_{0,0}(0)^{2} \theta_{0,0}(r-z)^{2}-\theta_{0,1}(0)^{2} \theta_{0,1}(r-z)^{2}-\theta_{1,0}(0)^{2} \theta_{1,0}(r-z)^{2}=0, \\
& \theta_{0,0}(0)^{2} \theta_{0,0}(r+z) \theta_{0,0}(r-z)-\theta_{0,1}(0)^{2} \theta_{0,1}(r+z) \theta_{0,1}(r-z)- \\
& \theta_{1,0}(0)^{2} \theta_{1,0}(r+z) \theta_{1,0}(r-z)=\theta_{1,1}(z)^{2} \theta_{1,1}(r)^{2}
\end{aligned}
$$

The equations (5.2) for other subsets $T$ produce other known identities for $\theta$ functions. In the case of generic $n$ the situation is similar: in parametrization (5.3) the equations (5.2) turn into certain special cases of the Riemann identities (Frobenius formulae [19]).

Returning to the integrable models we conclude the following: the formulae (5.3) provide the parametrization of $M(\lambda)$ on the torus ( Jacobian), that is why the times $t_{i}$ should be related to $z_{j}$ as

$$
t_{i}=\sum_{j=1}^{g} c_{i}^{j} z_{j}
$$

the constants $c_{i}^{j}$ refer to the particular integrable equation which is not essential for us.

Let us consider now the same situation from different point of view. What will follow is an extraction from Adler-Reyman-Semenov-Tian-Shansky approach[21,22]. Consider the loop algebra $\mathfrak{g}=\widehat{s l}(2)$ with generators $J_{m}^{a}, a=1,2,3 ; m$ is an integer. The generators satisfy the relations:

$$
\left[J_{m}^{a}, J_{n}^{b}\right]=f_{c}^{a, b} J_{n+m}^{c}
$$


The algebra contains two subalgebras: $\mathfrak{g}_{-}$generated by $J_{m}^{a}$ with $m<0$ and $\mathfrak{g}_{+}$ generated by $J_{m}^{a}$ with $m \geq 0$. Evidently, the algebra allows finite-dimensional representation $\rho_{\lambda}$ :

$$
\rho_{\lambda}\left(J_{m}^{a}\right)=\lambda^{m} \sigma^{a}
$$

By $G=\widehat{S L}(2)$ we denote a groop whose Lie algebra coincides with $\mathfrak{g}$. This group contains two subgroups:

$$
G_{+}=\exp \left(\mathfrak{g}_{+}\right), G_{-}=\exp \left(\mathfrak{g}_{-}\right)
$$

Consider now the matrix $M(\lambda)$ of the same type as above (traceless, polynomial in $\lambda$ of degree $n$ with fixed senior coefficient) and introduce the action of $G$ on $M(\lambda)$ as follows:

$$
g(M(\lambda))=\rho_{\lambda}(g) M(\lambda) \rho_{\lambda}(g)^{-1}, \text { for } g \in G
$$

The subgroups $G_{+}, G_{-}$acting on $M(\lambda)$ generate the orbits $O_{+}, O_{-}$of the form:

$$
\begin{aligned}
& g_{+}(M(\lambda))=\sum_{k=0}^{\infty} m_{k}^{\prime} \lambda^{k}, \\
& g_{-}(M(\lambda))=\sum_{k=-\infty}^{n} m_{k}^{\prime \prime} \lambda^{k}, m_{n}^{\prime \prime}=\sigma_{3}
\end{aligned}
$$

The algebraic interpretation of the integrable models is explained as the problem of description of the intersection $J$ of the orbits $O_{+}, O_{-}$. Clearly this intersection is composed of the matrix of the same type as $M(\lambda)$ itself, so the connection with the matters discussed above is manifest. How to describe the intersection in question in algebraic way? If $M^{\prime} \in J$ then there exist $g_{+}, g_{-}$such that

$$
\begin{aligned}
& g_{+}(M(\lambda))=\rho_{\lambda}\left(g_{+}\right) M(\lambda) \rho_{\lambda}\left(g_{+}\right)^{-1}= \\
& =g_{-}(M(\lambda))=\rho_{\lambda}\left(g_{-}\right) M(\lambda) \rho_{\lambda}\left(g_{-}\right)^{-1}
\end{aligned}
$$

It is evident from these equations that the matrix $g(\lambda)=\rho_{\lambda}\left(g_{-}\right)^{-1} \rho_{\lambda}\left(g_{+}\right)$commutes with $M(\lambda)$. The only possibility for the matrix to commute with $M(\lambda)$ which is supposed to be not degenerate for generic $\lambda$ is to be a function of $M(\lambda)$. Fortunately, additional dependence on $\lambda$ makes the situation nontrivial. We can consider $g(\lambda)$ of the form:

$$
g(\lambda)=\exp \left\{\sum t_{i} M_{i}(\lambda)\right\}
$$

where $M_{i}(\lambda)=\lambda^{-i} M(\lambda)$.

Now let us inverse the reasonings which means to start with $g(\lambda)$ in the form (5.6), and to try to construct $J$. Evidently to this end we need to solve the following Riemann problem: present

$$
g(\lambda)=\exp \left\{\sum t_{i} M_{i}(\lambda)\right\}=\rho_{\lambda}\left(g_{-}\right)^{-1} \rho_{\lambda}\left(g_{+}\right)
$$

where the Loran series for $\rho_{\lambda}\left(g_{+}\right),\left(\rho_{\lambda}\left(g_{-}\right)\right)$contain only positive (negative) powers. It is clear that only those $M_{i}$ are essential for which $0<i<n$, others can be directly moved to either $g_{+}$or $g_{-}$, and do not contribute to (5.5). So, we deal with the 
dependence on $n-1$ times which can be, actually, identified with the parameters on the Jacobian considered above.

Suppose the problem (5.7) is solved. Then we can introduce "times" dependent matrices $M\left(\lambda, t_{1}, \cdots, t_{g}\right)$ as being dressed according to (5.5) by $g_{+}$or $g_{-}$from (5.7). It easy to show that the projections of $\lambda^{-i} M\left(\lambda, t_{1}, \cdots, t_{g}\right)$ onto $\mathfrak{g}_{+}$denoted by $\mathfrak{M}_{i}$ satisfy the equations:

$$
\frac{\partial}{\partial t_{i}} \mathfrak{M}_{j}-\frac{\partial}{\partial t_{j}} \mathfrak{M}_{i}=\left[\mathfrak{M}_{i}, \mathfrak{M}_{j}\right]
$$

which coincide with (5.1).

We would like to emphasize the importance of the vectors $\psi_{i}$ from the algebraic point of view. The BA function itself does not transform in a reasonable way under the transformations (5.4), but its values at the branching points $\left(\psi_{i}\right)$ do transform under finite-dimensional representation of $G$ when $M$ transforms under (5.4):

$$
\psi_{i} \rightarrow G\left(\lambda_{i}\right) \psi_{i}
$$

This nice property of $\psi_{i}$ will be important in what follows.

To finish this section let us write down explicitly the equations in terms of $m_{i}^{a}$ :

$$
\frac{\partial}{\partial t_{k}} m_{j}^{a}=\sum_{q=\max (0, k+j-n)}^{j} f^{a b c} m_{j-q+k}^{b} m_{q}^{c}
$$




\section{Tau-function and $\mathrm{KZ}$ equations.}

The most mysterious object in the theory of classical integrable equations is that of $\tau$-function [23-26]. Hirota observed that the integrable classical equations can be rewritten as follows. Let us consider certain function $\tau$, depending upon the arguments $t=\left\{t_{1}, t_{2}, \cdots\right\}$ and introduce the notations:

$$
\left.D_{i_{1}} \cdots D_{i_{k}} \tau \cdot \tau(t) \equiv \frac{\partial}{\partial x_{i_{1}}} \cdots \frac{\partial}{\partial x_{i_{k}}} \tau(t+x) \tau(t-x)\right|_{x=0}
$$

Then according to Hirota the integrable equations can be written as

$$
P(D) \tau \cdot \tau=0
$$

for certain "polynomial" in $D$. We shall be interested mostly not in $\tau$-function itself, but in the linear space of functions $\mathfrak{V}$ which is defined as a space spanned by the functions $\left[D_{i_{1}} \cdots D_{i_{k}} \tau \cdot \tau(t)\right] / \tau(t)^{2}$. Hirota equations (6.1) imply certain linear dependence in the space.

The main achievement of Kyoto school in that direction is the formula expressing $\tau$-function in terms of vacuum expectation in the space of highest weight representation for the algebra $\widehat{s l}(2)$ on level 1 [24]. Consider the Cartan subalgebra generated by $I_{k} \equiv J_{k}^{3}, k>0$. Then the formula takes place

$$
\tau(t)=\left\langle\Omega\left|g \exp \left(\sum_{k} t_{k} I_{k}\right)\right| \Omega\right\rangle
$$

where $|\Omega\rangle$ is the highest vector of the representation on level $1,\langle\Omega|$ is the dual vector, $g$ is the element of central extended group $\widehat{S L}(2)$ which specifies a particular solution of the equation. We want to relate the $\mathrm{KZ}$ equations on level zero to $\tau$ function. The formula (6.2) is both good and bad for our goals. Good thing is that (6.2) links the integrable equations to the highest weight representations which are involved in KZ. Bad thing is that the formula (6.2) on the one hand and our KZ on another deal with different central extensions of $\widehat{s l}(2)$. We shall take the good thing as a hint of possible connection between $k=0 \mathrm{KZ}$ and $\tau$-function, and forget about the bad thing. It should be said also that for the case we are interested in (that of finite-gap integration) the description of $g$ is rather complicated and indirect.

What is $\tau$-function in the finite-gap case? The answer is well known: it is essentially the $\theta$-function $\theta[\delta](z), t_{i}=\sum_{k} c_{i}^{k} z_{k}$, where $c$ are some coefficients. How is it connected with BA-function? The usual answer is that it coincides with the value of BA-function at certain point $(\infty)$. This answer does not satisfy us because we do not like to use BA-function at generic point for its unclear algebraic properties, we want to deal with the values of BA-function at the branching points $\left(\psi_{j}\right)$ only.

The space $\mathfrak{V}$ is finite-dimensional in finite-gap case. Let us consider the example $g=1$. All the times $t_{i}$ are proportional to one variable on the Jacobian $z$. The $\tau$-function is $\theta_{1,1}(z)$. The space $\mathfrak{V}$ is generated by 1 and $\frac{D^{2} \tau \cdot \tau}{\tau^{2}}=\frac{\partial^{2}}{\partial z^{2}} \log \theta_{1,1}(z)$. The Hirota equation reads as

$$
D^{4} \tau \cdot \tau+c_{1} D^{2} \tau \cdot \tau+c_{2} \tau^{2}=0
$$


which turns into well-known differential equation for $\theta$-function (equivalent to the equation for the Weierestrass $\mathfrak{P}$-function):

$$
\frac{\partial^{4}}{\partial z^{4}} \log \theta_{1,1}(z)+6\left(\frac{\partial^{2}}{\partial z^{2}} \log \theta_{1,1}(z)\right)^{2}+c_{1} \frac{\partial^{2}}{\partial z^{2}} \log \theta_{1,1}(z)+c_{2}=0
$$

for some $c_{1}, c_{2}$.

Our further strategy will be in putting together the space $\mathfrak{V}$, the vectors $\psi_{i}$ and the solutions of $k=0 \mathrm{KZ}$ equations the idea being the following: $\psi_{i}$ transforms under finite-dimensional representation of $\widehat{s l}(2), \tau$ is connected with highest weight representation, hence they should be put together via KZ equations. To start let us consider the following object:

$$
v(z) \equiv \bar{\psi}_{1, \epsilon_{1}}(z) \cdots \bar{\psi}_{2 n, \epsilon_{2 n}}(z)\left\langle 0\left|V^{\epsilon_{1}}\left(\lambda_{1}\right) \cdots V^{\epsilon_{2 n}}\left(\lambda_{2 n}\right)\right| 0\right\rangle
$$

it should be said that $v(z)$ is not a function but rather a set of functions because different choices of blocks of vertex operators (intermediate Verma moduli) are possible. This is the same as different choices of cycles $A^{\prime}$ for $f_{A^{\prime}}\left(\lambda_{1}, \cdots, \lambda_{2 n}\right)^{\epsilon_{1}, \cdots, \epsilon_{2 n}}$ :

$$
\left\langle 0\left|V^{\epsilon_{1}}\left(\lambda_{1}\right) \cdots V^{\epsilon_{2 n}}\left(\lambda_{2 n}\right)\right| 0\right\rangle_{\text {some }} \quad \text { block }=f_{\text {some } A^{\prime}}\left(\lambda_{1}, \cdots, \lambda_{2 n}\right)^{\epsilon_{1}, \cdots, \epsilon_{2 n}}
$$

So, if necessary we shall indicate a particular component of $v$ as $v_{A^{\prime}}$. The first important property of the functions $v(z)$ is that their dependence on $z$ is governed by "free" dynamics in Verma module.

Let us take the matrix $M(\lambda)$ as described in the previous section:

$$
M(\lambda)=\sum_{i=0}^{n} \lambda^{i} m_{i}^{a} \sigma^{a}, m_{n}^{a}=\delta_{a, 3}, \operatorname{det}(M(\lambda))=\prod_{i=1}^{2 n}\left(\lambda-\lambda_{j}\right)
$$

Associate to the matrix $M(\lambda)$ the element of $\mathfrak{g}$

$$
\widehat{M}=\sum_{i=0}^{n} m_{i}^{a} J_{i}^{a}
$$

such that $M(\lambda)=\rho_{\lambda}(\widehat{M})$. Similarly, the algebra elements $\widehat{M}_{j}$ can be introduced:

$$
\widehat{M}_{j}=\sum_{i=0}^{n} m_{i}^{a} J_{i-j}^{a}, \quad \rho_{\lambda}\left(\widehat{M}_{j}\right)=M_{j}(\lambda)
$$

Clearly, $\widehat{M}_{j}$ commute among themselves:

$$
\left[\widehat{M}_{i}, \widehat{M}_{j}\right]=0
$$

Let us consider $M(\lambda)$ as a starting point for the procedure of the previous section, i.e. $M(\lambda)$ is the stationary $M$-operator corresponding to the moment $t_{i}=0$. To the matrix $M(\lambda)$ we attach the set of vectors $\psi_{i}, i=1, \cdots, 2 n$ as it is explained above. The following important statement holds. 
Proposition The $z$-dependence of $v(z)$ is governed by "free" dynamics in the Verma module:

$$
\begin{aligned}
& v(z)=\bar{\psi}_{1, \epsilon_{1}}(z) \cdots \bar{\psi}_{2 n, \epsilon_{2 n}}(z)\left\langle 0\left|V^{\epsilon_{1}}\left(\lambda_{1}\right) \cdots V^{\epsilon_{2 n}}\left(\lambda_{2 n}\right)\right| 0\right\rangle= \\
& \bar{\psi}_{1, \epsilon_{1}}(0) \cdots \bar{\psi}_{2 n, \epsilon_{2 n}}(0)\left\langle 0\left|V^{\epsilon_{1}}\left(\lambda_{1}\right) \cdots V^{\epsilon_{2 n}}\left(\lambda_{2 n}\right) \exp \left(\sum_{i=1}^{n-1} \widehat{M}_{i} t_{i}\right)\right| 0\right\rangle
\end{aligned}
$$

where $z$ and $t$ are connected by some linear transformation: $t_{i}=c_{i}^{j} z_{j}$.

In what follows we shall allow ourselves inaccuracy using both notations: $\psi_{i}(z)$ and $\psi_{i}(t)$ assuming that they coincide when $z$ and $t$ are properly related. As it was explained in the previous section the functions $\bar{\psi}_{i}$ satisfy the following equation:

$$
\frac{\partial}{\partial t_{k}} \bar{\psi}_{i}(t)=\bar{\psi}_{i}(t) \mathfrak{M}\left(\lambda_{i}, t\right)
$$

where the $t$-dependence of $\mathfrak{M}$ is due to (5.8). This equation allows to express all the higher derivatives of $\bar{\psi}$ at the moment $t=0$ in terms of derivatives of $\mathfrak{M}$. Let us prove the equations (6.3) comparing all the derivatives at $t=0$ of LHS and RHS.

With first derivative it is trivial, in LHS one has:

$$
\begin{aligned}
& \left.\frac{\partial}{\partial t_{i}}\left(\bar{\psi}_{1, \epsilon_{1}}(t) \cdots \bar{\psi}_{2 n, \epsilon_{2 n}}(t)\left\langle 0\left|V^{\epsilon_{1}}\left(\lambda_{1}\right) \cdots V^{\epsilon_{2 n}}\left(\lambda_{2 n}\right)\right| 0\right\rangle\right)\right|_{t=0}= \\
& =\sum_{p=1}^{2 n} \bar{\psi}_{1, \epsilon_{1}}(0) \cdots \bar{\psi}_{p-1, \epsilon_{p-1}}(0) \bar{\psi}_{p, \epsilon_{p}^{\prime}}(0) \bar{\psi}_{p+1, \epsilon_{p+1}}(0) \cdots \bar{\psi}_{2 n, \epsilon_{2 n}}(0) \\
& \times \mathfrak{M}_{i, \epsilon_{p}}^{\epsilon_{p}^{\prime}}\left(\lambda_{p}\right)\left\langle 0\left|V^{\epsilon_{1}}\left(\lambda_{1}\right) \cdots V^{\epsilon_{2 n}}\left(\lambda_{2 n}\right)\right| 0\right\rangle
\end{aligned}
$$

In RHS one has:

$$
\begin{aligned}
& \left.\frac{\partial}{\partial t_{i}} \bar{\psi}_{1, \epsilon_{1}}(0) \cdots \bar{\psi}_{2 n, \epsilon_{2 n}}(0)\left\langle 0\left|V^{\epsilon_{1}}\left(\lambda_{1}\right) \cdots V^{\epsilon_{2 n}}\left(\lambda_{2 n}\right) \exp \left(\sum_{i=1}^{n-1} \widehat{M}_{i} t_{i}\right)\right| 0\right\rangle\right|_{t=0}= \\
& =\bar{\psi}_{1, \epsilon_{1}}(0) \cdots \bar{\psi}_{2 n, \epsilon_{2 n}}(0)\left\langle 0\left|V^{\epsilon_{1}}\left(\lambda_{1}\right) \cdots V^{\epsilon_{2 n}}\left(\lambda_{2 n}\right) \widehat{M}_{i,+}\right| 0\right\rangle= \\
& =\sum_{p=1}^{2 n} \bar{\psi}_{1, \epsilon_{1}}(0) \cdots \bar{\psi}_{p-1, \epsilon_{p-1}}(0) \bar{\psi}_{p, \epsilon_{p}^{\prime}}(0) \bar{\psi}_{p+1, \epsilon_{p+1}}(0) \cdots \bar{\psi}_{2 n, \epsilon_{2 n}}(0) \\
& \times \mathfrak{M}_{i, \epsilon_{p}}^{\epsilon_{p}^{\prime}}\left(\lambda_{p}\right)\left\langle 0\left|V^{\epsilon_{1}}\left(\lambda_{1}\right) \cdots V^{\epsilon_{2 n}}\left(\lambda_{2 n}\right)\right| 0\right\rangle
\end{aligned}
$$

Evaluating the RHS we used the notation $\widehat{M}_{i,+}$ for the projection of $\widehat{M}_{i}$ onto $\mathfrak{g}_{+}$; recall that $J_{m}^{a}|0\rangle=0, m<0$. We moved $\widehat{M}_{i,+}$ to the left using the properties of the vertex operators, $\widehat{M}_{i,+}$ annihilates the left vacuum, also $\mathfrak{M}_{i}\left(\lambda_{p}\right)=\rho_{\lambda_{p}}\left(\widehat{M}_{i,+}\right)$. 
Less trivial computation is that for the second derivatives. In LHS one gets

$$
\begin{aligned}
& \left.\frac{\partial}{\partial t_{i}} \frac{\partial}{\partial t_{j}}\left(\bar{\psi}_{1, \epsilon_{1}}(t) \cdots \bar{\psi}_{2 n, \epsilon_{2 n}}(t)\left\langle 0\left|V^{\epsilon_{1}}\left(\lambda_{1}\right) \cdots V^{\epsilon_{2 n}}\left(\lambda_{2 n}\right)\right| 0\right\rangle\right)\right|_{t=0}= \\
& =\sum_{p<q}^{2 n} \bar{\psi}_{1, \epsilon_{1}}(0) \cdots \bar{\psi}_{p-1, \epsilon_{p-1}}(0) \bar{\psi}_{p, \epsilon_{p}^{\prime}}(0) \bar{\psi}_{p+1, \epsilon_{p+1}}(0) \cdots \\
& \times \bar{\psi}_{q-1, \epsilon_{q-1}}(0) \bar{\psi}_{q, \epsilon_{q}^{\prime}}(0) \bar{\psi}_{q+1, \epsilon_{q+1}}(0) \bar{\psi}_{2 n, \epsilon_{2 n}}(0) \\
& \times\left\{\mathfrak{M}_{i, \epsilon_{p}^{\prime \prime}}^{\epsilon_{p}^{\prime}}\left(\lambda_{p}\right) \mathfrak{M}_{j, \epsilon_{q}}^{\epsilon_{q}^{\prime \prime}}\left(\lambda_{q}\right)+\mathfrak{M}_{i, \epsilon_{q}^{\prime \prime}}^{\epsilon_{q}^{\prime}}\left(\lambda_{q}\right) \mathfrak{M}_{j, \epsilon_{p}}^{\epsilon_{p}^{\prime \prime}}\left(\lambda_{p}\right)\right\}\left\langle 0\left|V^{\epsilon_{1}}\left(\lambda_{1}\right) \cdots V^{\epsilon_{2 n}}\left(\lambda_{2 n}\right)\right| 0\right\rangle+ \\
& +\sum_{p=1}^{2 n} \bar{\psi}_{1, \epsilon_{1}}(0) \cdots \bar{\psi}_{p-1, \epsilon_{p-1}}(0) \bar{\psi}_{p, \epsilon_{p}^{\prime}}(0) \bar{\psi}_{p+1, \epsilon_{p+1}}(0) \cdots \bar{\psi}_{2 n, \epsilon_{2 n}}(0) \\
& \times\left\{\mathfrak{M}_{i, \epsilon_{p}^{\prime \prime}}^{\epsilon_{p}^{\prime}}\left(\lambda_{p}\right) \mathfrak{M}_{j, \epsilon_{p}}^{\epsilon_{p}^{\prime \prime}}\left(\lambda_{p}\right)+\partial_{i} \mathfrak{M}_{j, \epsilon_{p}}^{\epsilon_{p}^{\prime}}\left(\lambda_{p}\right)\right\}\left\langle 0\left|V^{\epsilon_{1}}\left(\lambda_{1}\right) \cdots V^{\epsilon_{2 n}}\left(\lambda_{2 n}\right)\right| 0\right\rangle
\end{aligned}
$$

To transform the RHS one has to deal with $\widehat{M}_{i} \widehat{M}_{j}|0\rangle$ which can be evaluated due to the equations (5.8):

$$
\begin{aligned}
& \widehat{M}_{i} \widehat{M}_{j}|0\rangle=\left(\widehat{M}_{i,+}+\widehat{M}_{i,-}\right) \widehat{M}_{j}|0\rangle= \\
& =\widehat{M}_{i,+} \widehat{M}_{j,+}|0\rangle+\partial_{i} \widehat{M}_{j,+}|0\rangle
\end{aligned}
$$

Now moving $\widehat{M}_{i,+} \widehat{M}_{j,+}$ and $\partial_{i} \widehat{M}_{j,+}$ to the left one gets (6.4). The consideration of general case is quite similar to the case of the second derivative which is the most demonstrative one.

Thus, we have shown that the functions $v(z)$ possess the nice property (6.3) which makes them similar to $\tau$-function.

It should be mentioned that the starting $M(\lambda)$ can be taken in especially simple way. For example we can divide the set of $\lambda_{i}$ into two subsets $\left(B=S \cup S^{\prime}\right.$, \#S= $\left.\# S^{\prime}=n\right)$ and define $M$ as follows:

$$
M(\lambda)=\left(\begin{array}{cc}
\prod_{i \in S}\left(\lambda-\lambda_{i}\right)+\prod_{i \in S^{\prime}}\left(\lambda-\lambda_{i}\right), & \prod_{i \in S}\left(\lambda-\lambda_{i}\right)-\prod_{i \in S^{\prime}}\left(\lambda-\lambda_{i}\right) \\
\prod_{i \in S^{\prime}}\left(\lambda-\lambda_{i}\right)-\prod_{i \in S}\left(\lambda-\lambda_{i}\right), & -\prod_{i \in S}\left(\lambda-\lambda_{i}\right)-\prod_{i \in S^{\prime}}\left(\lambda-\lambda_{i}\right)
\end{array}\right)
$$

In that case the vectors $\psi_{i}$ are also simple.

We have to connect the functions $v(z)$ with some familiar objects. There should be a clever way to get the result which will be announced soon. But we do not know that way which makes us to proceed to calculations. Let us consider the elliptic case. Recall that the covectors $\bar{\psi}_{i}$ are given in the elliptic case by:

$$
\begin{aligned}
& \bar{\psi}_{1}(z) \simeq\left(\frac{\theta_{0,0}(r+z)}{\theta_{1,1}(z)}, \frac{\theta_{0,0}(r-z)}{\theta_{1,1}(z)}\right) \\
& \bar{\psi}_{2}(z) \simeq\left(\frac{\theta_{0,1}(r+z)}{\theta_{1,1}(z)}, \frac{\theta_{0,1}(r-z)}{\theta_{1,1}(z)}\right), \\
& \bar{\psi}_{3}(z) \simeq\left(\frac{\theta_{1,1}(r+z)}{\theta_{1,1}(z)}, \frac{\theta_{1,1}(r-z)}{\theta_{1,1}(z)}\right) \\
& \bar{\psi}_{4}(z) \simeq\left(\frac{\theta_{1,0}(r+z)}{\theta_{1,1}(z)}, \frac{\theta_{1,0}(r-z)}{\theta_{1,1}(z)}\right) \\
& 27
\end{aligned}
$$


In what follows we shall perform calculations up to constants which will be outlined by using $\simeq$. There are two solutions to KZ equations in elliptic case which are given by are given by

$$
\begin{aligned}
& f_{a}\left(\lambda_{1}, \cdots, \lambda_{4}\right)^{\epsilon_{1}, \cdots, \epsilon_{4}} \simeq \theta\left[\eta_{T}\right](0)^{2} \partial^{2} \theta\left[\eta_{T}\right](0)^{2} \\
& f_{a^{\prime}}\left(\lambda_{1}, \cdots, \lambda_{4}\right)^{\epsilon_{1}, \cdots, \epsilon_{4}} \simeq \theta\left[\eta_{T}\right](0)^{2}\left[K \partial^{2}+1\right] \theta\left[\eta_{T}\right](0)^{2}
\end{aligned}
$$

the correspondence between $\epsilon_{1}, \cdots, \epsilon_{4}, T$ and conventional notations for elliptic $\theta$-functions is given by (4.19). This formulae suggest to generalize the expression for $v(z)$ substituting in it $\theta\left[\eta_{T}\right](0)^{2} \theta\left[\eta_{T}\right](x)^{2}$ instead of the solutions of KZ. Doing that one gets the following:

$$
\begin{aligned}
& v(z, x) \simeq \theta_{1,1}(z)^{-4} \\
& \times\left[\theta _ { 1 , 0 } ( 0 ) ^ { 2 } \theta _ { 1 , 0 } ( x ) ^ { 2 } \left\{\theta_{0,0}(r+z) \theta_{0,1}(r+z) \theta_{1,1}(r-z) \theta_{1,0}(r-z)+\right.\right. \\
& \left.+\theta_{0,0}(r-z) \theta_{0,1}(r-z) \theta_{1,1}(r+z) \theta_{1,0}(r+z)\right\}+ \\
& +\theta_{0,1}(0)^{2} \theta_{0,1}(x)^{2}\left\{\theta_{0,0}(r+z) \theta_{0,1}(r-z) \theta_{1,1}(r-z) \theta_{1,0}(r+z)+\right. \\
& \left.+\theta_{0,0}(r-z) \theta_{0,1}(r+z) \theta_{1,1}(r+z) \theta_{1,0}(r-z)\right\}+ \\
& +\theta_{0,0}(0)^{2} \theta_{0,0}(x)^{2}\left\{\theta_{0,0}(r+z) \theta_{0,1}(r-z) \theta_{1,1}(r+z) \theta_{1,0}(r-z)+\right. \\
& \left.\left.+\theta_{0,0}(r-z) \theta_{0,1}(r+z) \theta_{1,1}(r-z) \theta_{1,0}(r+z)\right\}\right]
\end{aligned}
$$

This expression can be simplified via Riemann identities the result being quite simple:

$$
v(z, x) \simeq \frac{\theta_{1,1}(z+x) \theta_{1,1}(z-x)}{\theta_{1,1}(z)^{2}}
$$

which leads to the following nice formulae for $v_{a}(z), v_{a^{\prime}}(z)$ :

$$
v_{a}(z) \simeq \frac{D^{2} \tau \cdot \tau}{\tau^{2}}(z), \quad v_{a^{\prime}}(z) \simeq K \frac{D^{2} \tau \cdot \tau}{\tau^{2}}(z)+1
$$

where $\theta_{1,1}$ is denoted by $\tau, D$ is Hirota derivative.

Thus by convoluting $\bar{\psi}_{i}$ and the solutions of KZ in the elliptic case we got exactly the basis of the space $\mathfrak{V}$. Combining that with the proposition above we also realize that the dynamics in the space $\mathfrak{V}$ is governed by the free dynamics in the Verma module. We would suppose that this nice connection holds in generic case as well. In order to prove that we have to use the Conjecture 1 from Section 4 together with the following

Conjecture 2. The following identity (up to neglectable constants) for the $\theta$-functions on HES holds:

$$
\begin{aligned}
\sum_{T \subset B, \# T=g+1} \theta\left[\eta_{T}\right](0)^{2} \theta\left[\eta_{T}\right](x)^{2} \prod_{j=1}^{2 g+2} \theta\left[\eta_{j}\right]\left(r+\epsilon_{j} z\right) \simeq \\
\simeq \theta[\delta](z)^{2 g} \theta[\delta](z+x) \theta[\delta](z-x)
\end{aligned}
$$

where $T$ and $\left\{\epsilon_{i}\right\}$ are connected in usual way.

The author strongly believes in this conjecture, still he has been unable to prove it. 
If the conjectures 1,2 are both true in generic case then the following relation should take place:

$$
\mathfrak{V} \ni \frac{Q_{A^{\prime}} \tau \cdot \tau}{\tau^{2}}=\bar{\psi}_{1, \epsilon_{1}} \cdots \bar{\psi}_{2 n, \epsilon_{2 n}} f_{A^{\prime}}\left(\lambda_{1}, \cdots, \lambda_{2 n}\right)^{\epsilon_{1}, \cdots, \epsilon_{2 n}}
$$

where $\tau(z)=\theta[\delta](z)$. We would suppose also that there are enough $\theta$ - constants hidden in $f_{A^{\prime}}$, i.e. that all the independent Hirota derivatives of $\tau$ can be obtained by linear combinations of (6.5) for different $A^{\prime}$.

The formula (6.5) establishes the relation between $\bar{\psi}_{i}$ which transform under finite-dimensional representations of $\widehat{s l}(2)$ and the space of Hirota derivatives of $\tau$-function (the space of classical fields) via the solutions of $\mathrm{KZ}$ equations on level 0 which we can call classical form factors. This is exactly the formula we were looking for, its similarity with quantum formula (1.1) is manifest.

To finish this section we would like to present one more interesting formula:

$$
\begin{aligned}
& \left\langle 0\left|V^{\epsilon_{1}}\left(\lambda_{1}\right) \cdots V^{\epsilon_{2 n}}\left(\lambda_{2 n}\right) \widehat{M}^{2 n} \exp \left(\sum t_{i} \widehat{M}_{i}\right)\right| 0\right\rangle= \\
& =\psi_{1}^{\epsilon_{1}}(0) \cdots \psi_{2 n}^{\epsilon_{2 n}}(0)\left\{\left.\bar{\psi}_{1, \epsilon_{1}^{\prime}}(t) \cdots \bar{\psi}_{2 n, \epsilon_{2 n}^{\prime}}(t)\left\langle 0\left|V^{\epsilon_{1}^{\prime}}\left(\lambda_{1}\right) \cdots V^{\epsilon_{2 n}^{\prime}}\left(\lambda_{2 n}\right)\right| 0\right\rangle\right|_{t=0}\right\}= \\
& =\psi_{1}^{\epsilon_{1}}(0) \cdots \psi_{2 n}^{\epsilon_{2 n}}(0) v(t)
\end{aligned}
$$

This formula is due to the fact that

$$
\rho_{\lambda_{i}}(\widehat{M})^{2}=\left(\psi_{i} \otimes \bar{\psi}_{i}\right)^{2}=0
$$

Notice that as usual $\widehat{M}$ and $\lambda_{1}, \cdots, \lambda_{2 n}$ must be connected: $\operatorname{det}\left(\rho_{\lambda_{i}}(\widehat{M})\right)=$ $\prod\left(\lambda-\lambda_{i}\right)$. 


\section{Conclusions.}

Let us return to the very beginning of the paper. We were wondering whether a formula similar to (1.1) exists in classics. We have answered this question in the last section of the paper. Really, the formula (6.5) is very much similar to (1.1). It connects classical local fields (Hirota derivatives of $\tau$-function) with the tensor product of finite-dimensional representations of tha affine algebra via the classical form factors (the solutions of $\mathrm{KZ}$ on level 0 ). So, the similarity of the formulae (1.1) and (6.5) is described by:

$$
\begin{aligned}
\psi_{i}, \bar{\psi}_{i} & \leftrightarrow Z\left(\beta_{i}\right), Z^{*}\left(\beta_{i}\right) \\
\mathfrak{V} & \leftrightarrow \mathfrak{A} \\
f & \leftrightarrow F
\end{aligned}
$$

The quantum formula needs also the summation over all particle states which can be interpreted as summation over all HES, the number of particles being the related to genus of surface, the rapidity being considered as the positions of branching points, i.e. the moduli of the surfaces. Notice that in the classical limit the difference between the finite- dimensional representation and conjugated one (which were responsible for particles and antiparticles in the quantum case) essentially disappears.

So, we would suppose the following procedure of quantization to be possible. We start with the family of finite-gap solutions of integrable model with $\widehat{s l}(2)$ symmetry. Different solutions are parametrized by the intersections of the orbits of $\mathfrak{g}_{ \pm}$as it is explained in the Section 5 . These intersections are parametrized by the matrix $M(\lambda)$, but we prefer to parametrize them by the set $\left\{\psi_{i}\right\}$ connected with $M(\lambda)$ as it is explained above. The vectors $\psi_{i}$ transform under finite-dimensional representation of the affine algebra. We can take

$$
H_{c l}=\bigoplus_{n=1}^{\infty} \int_{\lambda_{1}<\cdots<\lambda_{2 n}} V_{\lambda_{1}} \otimes \cdots \otimes V_{\lambda_{2 n}}
$$

( $V_{\lambda}$ is the space of the representation $\rho_{\lambda}$ ) as a completion of the manifold of classical finite-gap solutions. Not every vector from this space is good for the classical solutions but only those which can be presented in the form $\psi_{1} \otimes \cdots \otimes \psi_{2 n}$ with the set $\left\{\psi_{i}\right\}$ associated to a matrix $M(\lambda)$ as described above. For every particular element of this form the classical fields are given by convolution with classical form factor. In fact the space (7.1) coincides with the space of states of quantum model $H_{p}$ (1.3). Also the quantum form factor is a quantization of the classical one which is essentially due to the quantization of the algebra underlying the theory $(\widehat{s l}(2) \rightarrow D(Y))$. So, we propose that the following way for quantization should be possible: we extend the set of classical solutions to the space $H_{c l}$ and identify it with the space of states of quantum model, the rest of quantization is in quantizing of symmetry algebra. It should be strongly emphasized, however, that the space-time of the quantum model has nothing to do with the classical "times" $t_{i}$, it appears as a result of quantization as it is explained in Section 3.

Let us finish with several remarks. The finite-gap solution constitute in the classical theory nice but small subset of solutions. Generally, there are infinitegap solutions in which the finite-gap ones are of measure zero. The infinite-gap 
solutions are rather ugly ones, no reasonable theory is available for them. So, since for the quantization only finite-gap solutions are needed, we have a nice example of usual phenomena: quantization takes everything good from the classical theory and forgets about bad things typical for it. There is a puzzling connection between the matters discussed in the present paper and those considered in [27] in the connection with the theory of strings (this remark is due to H.Oogury). The constructions of the present paper can be generalized to $s u(N)$ case for which the algebra $\widehat{s l}(N)$ and the Riemann surfaces with the branching points of $N$-th order are responsible, the form factors of corresponding $s u(N)$-chiral Gross-Neveu are given in [11]. But probably this is not the best way for the generalization. We would better proceed to the consideration of arbitrary Riemann surfaces which are associated to KPequation. This case should cover all the $s u(N)$-invariant Gross-Neveu models and, probably, will lead to something essentially new.

Acknolegement. The work was partly done under Institute of Physics Fellowship at I.Newton Institute, Cambridge. The discussions with I.Cherednik, T.Eguchi, L.Faddeev, A.Kirillov, T.Miwa, H.Oogury, M.A. Semenov-Tian-Shansky were helpful. 


\section{References}

1. M. Lüscher, Nucl. Phys. B 135 (1978), 1

2. D. Bernard, CMP 137 (1991), 191

3. D. Bernard, A. LeClair, CMP 142 (1991), 99

4. G. Felder, A. LeClair, IJMPA 7, suppl.1 (1992),

5. A. LeClair, F.A. Smirnov, IJMPA 7 (1992),2997

6. F.A. Smirnov, IJMPA 7, suppl.2 (1992)

7. I.B. Frenkel, N.Yu. Reshetikhin, CMP 146 (1992), 1

8. L.D. Faddeev, E.K. Sklyanin, L.A. Takhtajan, Teor.Math.Phys. 40 (1980), 688

9. M. Jimbo, K. Miki, T. Miwa, A. Nakanishiki, Correlation functions of XXZ model for $\Delta<-1$ RIMS preprint 887 (1992)

10. A.B. Zamolodchikov. Al.B. Zamolodchikov, Annals of Physics 120 (1979), 253

11. F.A. Smirnov, Form Factors in Completely Integrable Models of Quantum Field Theory, Adv. Series in Math. Phys. 14, World Scientific (1992)

12. V.G. Drinfeld, Sov. Math. Dokl. 32 (1985) 254.

V.G. Drinfeld, Quantum Groups, Proceedings of the International Congress of Mathematicians, Berkeley, CA, (1987) 798

13. F.A. Smirnov, Remarks on deformed and undeformed KZ equations, RIMS preprint$860(1992)$

14. V.V. Schechtman, A.N. Varchenko, Integral Representations of $N$-point Conformal Correlations in the WZW Model, Max-Plank-Institute preprint (1987)

15. A. Tsuchiya, Y. Kanie, In: Conformal Field Theory and Solvable Lattice Models, Adv. Stud. Pure. Math. 16 (1988) 297.

16. A.R. Its, V.B. Matveev, Teor.Math.Phys. 23 (1975), 51

17. B.A.Dubrovin, V.B. Matveev, S.P. Novikov, Russian Math. Surveys 31, (1976) 59

18. I.M. Krichever, Russian Math. Surveys 32, (1977), 185

19. D. Mumford, Tata Lectures on Theta 1,2, Birkhäuser (1983)

20. J.D. Fay, Riemann functions on Riemann surfaces, Lecture Notes in Mathematics $352(1973)$

21. M.A. Semenov-Tian-Shansky, Publ.RIMS Kyoto Univ. 21 (1985), 1237

22. L.D. Faddeev, L.A. Takhtajan, Hamiltonian methods in the theory of solitons, Springer, (1987)

23. R. Hirota, in: Lecture Notes in Mathematics, 515, Springer (1976)

24. E. Date, M. Jimbo, M. Kashiwara, T. Miwa, in: Nonlinear Integrable Systems, World Scientific (1983)

25. G. Segal, G. Wilson, Publ. I.H.E.S. 61 (1985), 1

26. I. Cherednik, Funk. Anal. i Prilozh. 17 (1983), 93

27. V.G. Knizhnik, CMP 112 (1987), 567 